\title{
Critical Processes and Parameters in the Development of Accident Tolerant Fuels Drop-In Capsule Irradiation Tests
}

\author{
K. E. Barrett ${ }^{1}$, K. D. Ellis ${ }^{1}$, C. R. Glass ${ }^{1}$, G. A. Roth ${ }^{2}$, M. P. Teague ${ }^{1}$, J. Johns ${ }^{3}$ \\ 1. Idaho National Laboratory, P.O. Box 1625, Idaho Falls, ID, 83415, Kristine.Barrett@inl.gov \\ 2. Information Systems Laboratory, 2235 East $25^{\text {th }}$ St., Suite 100, Idaho Falls, ID 83404, groth@islinc.com \\ 3. Texas A\&M, College Station, TX 77843 jesse.m.johns@gmail.com.
}

\begin{abstract}
The goal of the Accident Tolerant Fuel (ATF) program is to develop the next generation of Light Water Reactor (LWR) fuels with improved performance, reliability, and safety characteristics during normal operations and accident conditions and with reduced waste generation. An irradiation test series has been defined to assess the performance of proposed ATF concepts under normal LWR operating conditions. The Phase I ATF irradiation test series is planned to be performed as a series of drop-in capsule tests to be irradiated in the Advanced Test Reactor (ATR) operated by the Idaho National Laboratory (INL).

Design, analysis, and fabrication processes for ATR drop-in capsule experiment preparation are presented in this paper to demonstrate the importance of special design considerations, parameter sensitivity analysis, and precise fabrication and inspection techniques for iFigurennovative materials used in ATF experiment assemblies. A Taylor Series Method sensitivity analysis approach was used to identify the most critical variables in cladding and rodlet stress, temperature, and pressure calculations for design analyses. The results showed that internal rodlet pressure calculations are most sensitive to the fission gas release rate uncertainty while temperature calculations are most sensitive to cladding I.D. and O.D. dimensional uncertainty. The analysis showed that stress calculations are most sensitive to rodlet internal pressure uncertainties, however the results also indicated that the inside radius, outside radius, and internal pressure were all magnified as they propagate through the stress equation.

This paper demonstrates the importance for ATF concept development teams to provide the fabricators as much information as possible about the material properties and behavior observed in prototype testing, mock-up fabrication and assembly, and chemical and mechanical testing of the materials that may have been performed in the concept development phase. Special handling, machining, welding, and inspection of materials, if known, should also be communicated to the experiment fabrication and inspection team.
\end{abstract}

\section{INTRODUCTION}

Until the unfortunate events at Fukushima, the emphasis of light water reactor (LWR) fuel development Research and Development (R\&D) activities for the U.S. Department of Energy - Office of Nuclear Energy (DOE-NE) was on improving the fuel performance in terms of increased burnup for waste minimization and increased power density for power upgrades, as well as collaborating with industry on fuel reliability. After the Fukushima events in 2011, the emphasis shifted to accident performance of the fuels under extended loss of active cooling and steam exposure. Subsequently, the congressional appropriation language for FY 2012 included specific language for DOE-NE to initiate an aggressive Research, Development, and Demonstration (RD\&D) program for LWR fuels with enhanced accident tolerance. As a result, DOE-NE has established a program for the development of LWR fuels with enhanced accident tolerance under the Fuel Cycle Research and Development (FCRD) Advanced Fuels Campaign (AFC) [1].

The goal of the Accident Tolerant Fuel (ATF) program is to develop the next generation of LWR fuels with improved performance, reliability, and safety characteristics during normal operations and accident conditions and with reduced waste generation. Enhancing the accident tolerance of the fuel is the focal point of the initiative. The initial RD\&D efforts are to focus on applications in operating reactors or reactors with design certifications. However, what is learned and developed during this process may be applicable to the design of the next generation of LWRs. 
As shown in Figure 1, the goal of the DOE ATF development program is to support the insertion of lead fuel rods (LFRs) or lead fuel assemblies (LFAs) in a commercial LWR within 10 years (Figure 1).

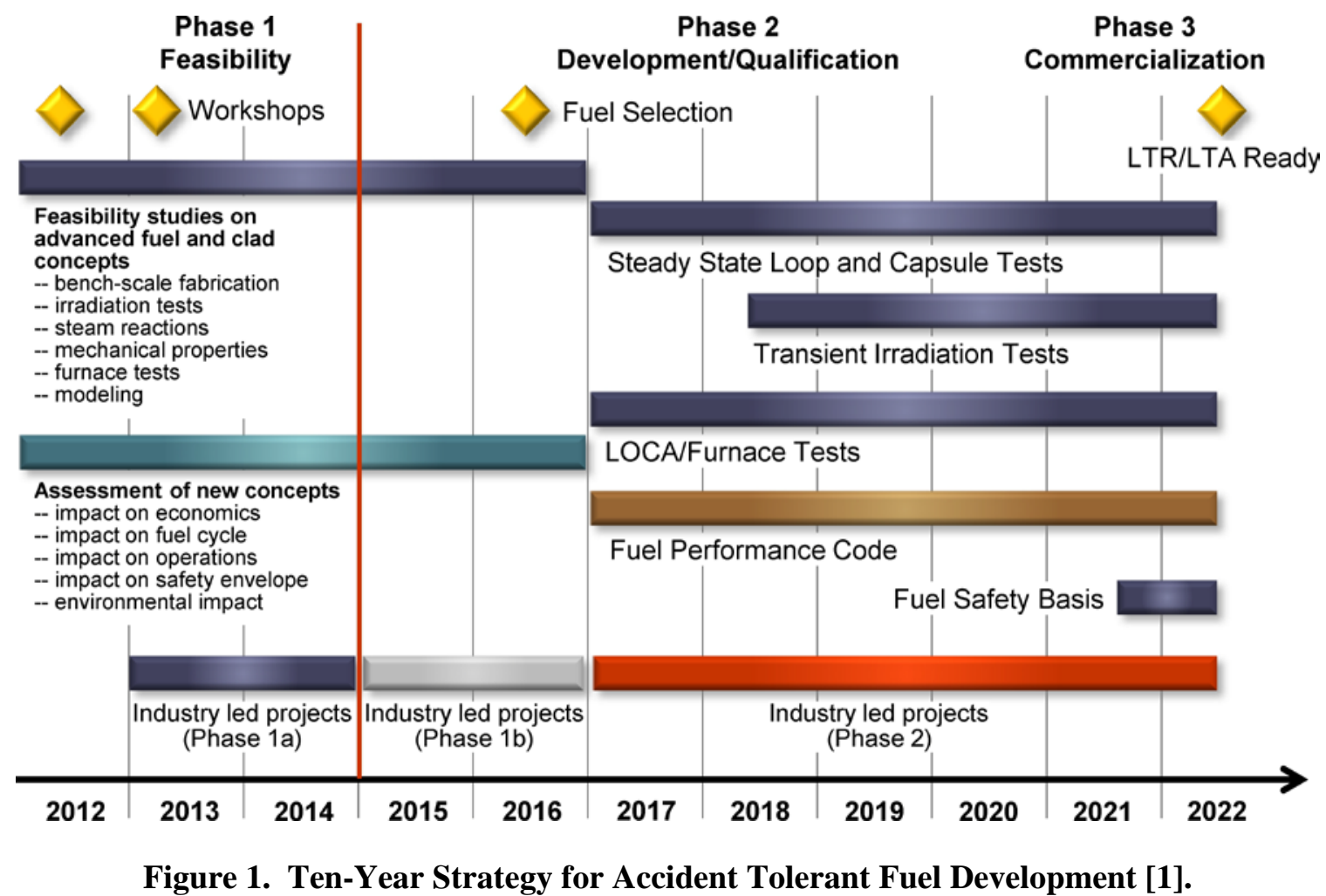

As a step toward these test goals, an irradiation test series has been defined to assess the performance of proposed ATF concepts under normal LWR operating conditions. Data generated by this test program will be used to establish the feasibility of certain aspects of proposed ATF concepts, as well as provide information to support screening among concepts; as such, it is an integral part of "Phase I: Feasibility Assessment and Down-Selection" outlined in the ATF Roadmap. This Phase I irradiation test series is planned to be performed as a series of drop-in capsule tests to be irradiated in the Advanced Test Reactor (ATR) operated by the Idaho National Laboratory (INL), and it has been designated as the ATF-1 Test Series [2].

The ultimate goal of the ATF Program is to demonstrate the performance of proposed fuel and/or cladding concepts that could replace the Zircaloy - $\mathrm{UO}_{2}$ system currently used throughout the LWR industry. The ATF-1 irradiation test series is a necessary step toward such a demonstration. Input has been solicited from ATF Development Teams concerning their ATF concept(s), appropriateness and desire to be included in the ATF-1 irradiation test series, and (where appropriate) tentative schedule for fabrication of test articles.

The ATF-1 Development Teams include the following:

1) Three campaign-directed efforts. The development of the Fully Ceramic Micro-encapsulated (FCM) fuel concept is led by Oak Ridge National Laboratory (ORNL). The development of the LWR U-Mo fuel concept is led by Pacific Northwest National Laboratory (PNNL). The development of UN/15 $\mathrm{U}_{3} \mathrm{Si}_{5}$ is led by Los Alamos National Laboratory. In addition, Los Alamos National Laboratory is a key contributor in the development of advanced, corrosion-resistant metallic alloys that could be used as cladding in a number of other ATF concepts.

2) Three Funding Opportunity Announcement (FOA) efforts. These FOA consortia are industry-led teams that also include DOE laboratories and universities as partners. The three FOA teams are led by Westinghouse, AREVA, and General Electric. 
3) Three Integrated Research Proposal (IRP) efforts. These IRP consortia are university-led teams involving multiple universities, and in some cases industry entities and/or DOE laboratories, as partners. The three IRP teams are led by the University of Illinois, University of Tennessee, and Georgia Institute of Technology. Two IRP teams (University of Illinois and University of Tennessee) are developing ATF cladding concepts but the third (Georgia Institute of Technology) is developing an Accident Tolerant (AT) reactor design and will likely use an ATF concept selected for Phase II and beyond testing.

The complex multiphysics behavior of LWR nuclear fuel systems makes defining specific material or design improvements difficult. Establishing quantitative attributes is critical to guide the design and development of fuels and cladding with enhanced accident tolerance. A set of technical evaluation metrics has been established and is included in the "Advanced Fuels Campaign Light Water Reactor Accident Tolerant Fuel Performance Metrics" report [3] to aid in the optimization and down-selection / prioritization of candidate designs. The assessment process is outlined in Figure 2. The resultant ranked evaluation can then inform concept down-selection, such that the most promising accident tolerant fuel design option(s) can continue to be developed for LFR or LFA insertion into a commercial reactor within the desired timeframe (by 2022).

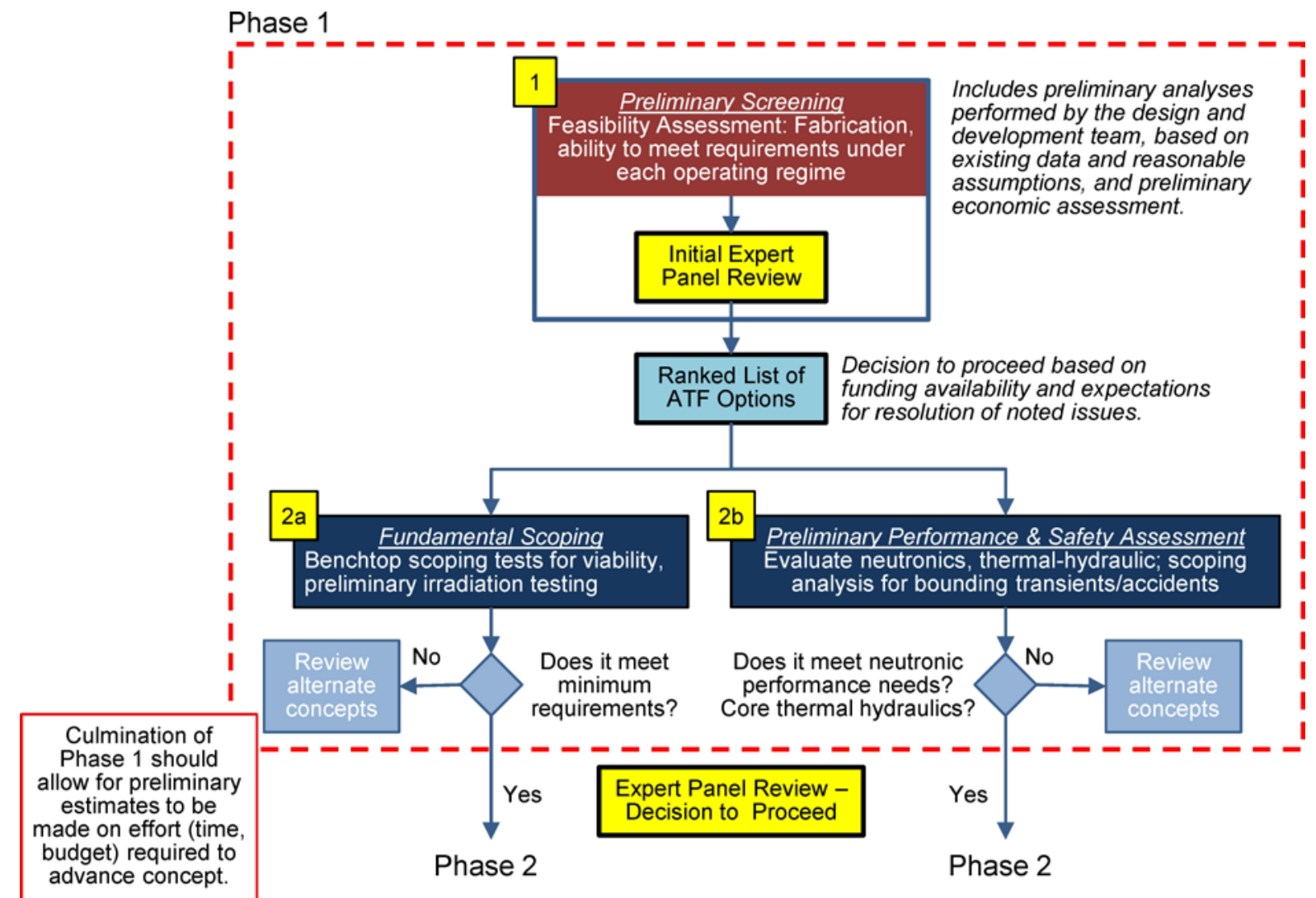

Figure 2. Phase I Feasibility Testing and Concept Selection Process for Phase II Continued Testing [1].

\section{PURPOSE / SCOPE}

The Idaho National Laboratory (INL) has the responsibility to conduct irradiation experiments in the Advanced Test Reactor (ATR) to assess the performance of ATF concepts under normal LWR operating conditions. INL is leading the planning, design, and analysis of these irradiation experiments in coordination with the various institutions that are engaged in developing ATF concepts. INL will perform these irradiation experiments in ATR and will coordinate the post-irradiation examinations (PIE) on the discharged fuels. 
For the first phase of in-reactor testing (ATF-1), the fuel/cladding system is placed inside a pressure boundary capsule to meet ATR safety requirements. This capsule isolates the experimental fuel rodlet from the reactor cooling water and provides the pressure boundary between the water and fuel. This capsule is then placed in the test reactor at a location that has a neutron fluence rate suitable to meet test design objectives. Before placing the fuel/cladding system in the ATR, a series of analyses must be performed to estimate the material performance and demonstrate a reactor safety margin. The flow diagram for this process is shown in Figure 3.

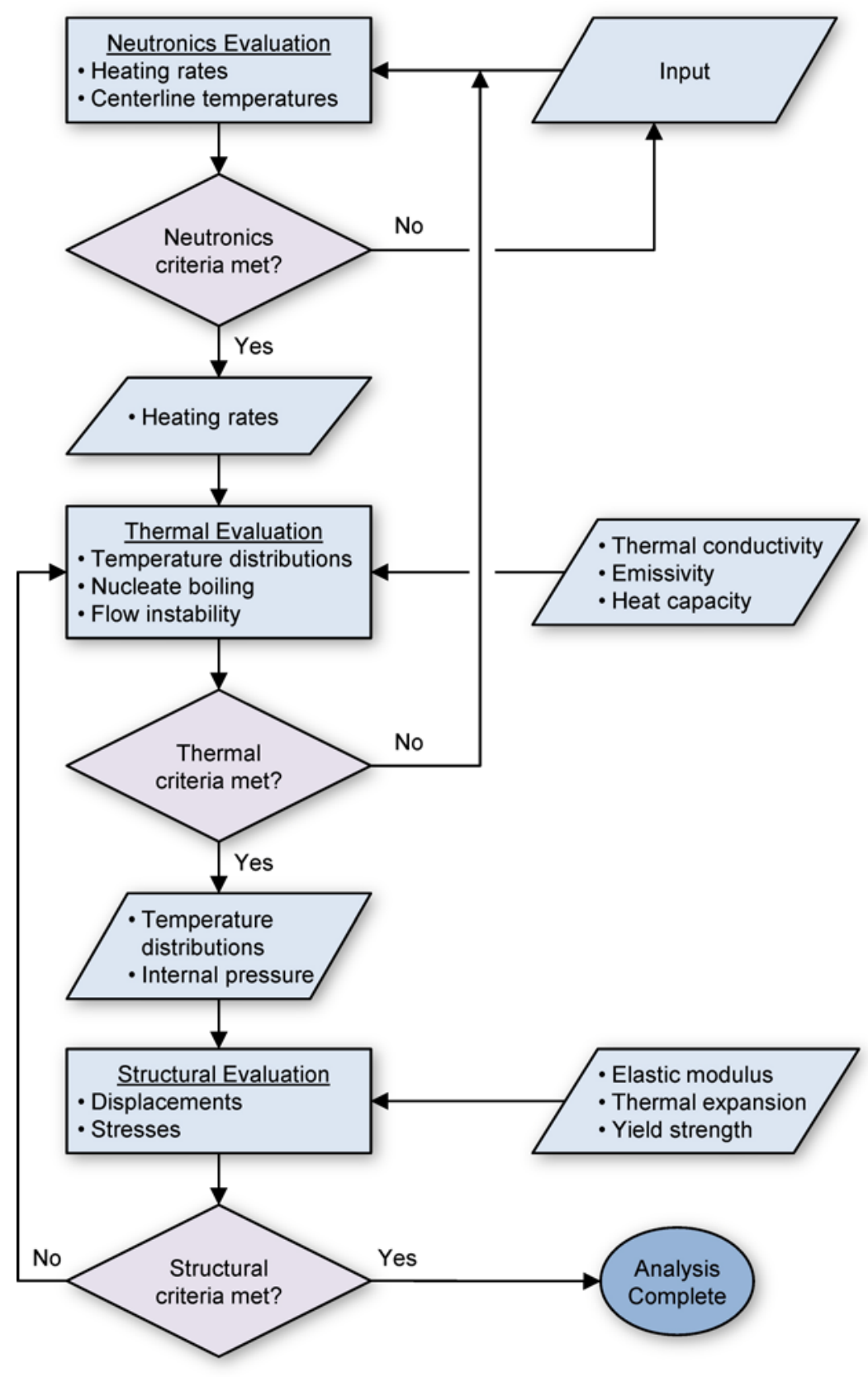

Figure 3. ATR Experiment Design Analysis Flow Chart.

There are many challenges in the design, development, and testing of test articles for ATR irradiation. These challenges are amplified for "drop-in" experiments where the test rodlet is encapsulated inside a pressure boundary capsule. The capsule provides two functions. First, it acts as a robust barrier between the primary reactor coolant and the experimental rodlet. 
Since the rodlet materials are often unproven, a potential rodlet breach during irradiation cannot be precluded. Therefore, secondary containment is required to ensure that fuel/fission products cannot be released into the primary reactor coolant. Second, the inert gas-filled gap between the capsule inner wall and rodlet outer wall introduces a thermal resistance that is necessary to elevate the rodlet cladding temperature to a level that is prototypic of LWR cladding. Prototypic temperatures cannot be achieved if the rodlet is cooled directly by the coolant. This dual fuel encapsulation design introduces design analyses and fabrication challenges in reaching and maintaining the test objective cladding temperature, in particular for fuel/cladding systems that include innovative materials with very little irradiation performance data to support analyses. Inreactor testing is one of the primary mechanisms available to evaluate the performance of these advanced fuel cladding systems; therefore, identifying the most sensitive parameter or set of parameters in the analyses is essential for evaluating analysis uncertainties and required experiment fabrication tolerances.

The focus of this paper is to describe a sensitivity analysis approach to identify the most sensitive beginning of life (BOL), i.e., pre-irradiated, variables in the performance of ATF fuel system concepts. Since the degree of uncertainty in the design analysis is difficult to quantify, the sensitivity analysis approach described in this paper provides design engineers with a more informed approach to select BOL variables and irradiation conditions that should be controlled as close to nominal as possible in the test. The premise of the ATF research is to determine the fuel system irradiation-induced properties, such as thermal expansion coefficients, creep, densification, fission gas release rates, and radiation swelling, which are for the most part unknown for the innovative ATF materials being tested. Although those unknown variables will no doubt impact the fuel system performance, understanding which known variables have the most impact on the fuel system parameter calculations allows design engineers and analysts to focus on these variables in the design and analysis process.

This paper outlines the design and analyses process for ATR drop-in capsule experiment preparation. It describes a Taylor Series Approach sensitivity analysis to identify the most sensitive parameters in BOL design analyses of ATF fuel system concepts. This paper also includes a discussion on fabrication challenges for meeting tight dimensional tolerances identified in the sensitivity analyses and the confirmatory "as-built" analyses performed to validate that the fabricated test articles meet test objectives prior to irradiation testing.

\section{TEST DESCRIPTION}

The irradiation test assembly consists of an aluminum experiment basket, inside of which are 316L stainless steel capsule(s) that serve as the primary safety (i.e., pressure) boundary, each of which encapsulates a single test rodlet made of fuel/material test specimens inside cladding. The experiment basket is designed to hold multiple, vertically stacked capsule assemblies. Each capsule assembly is independent, meaning it can be introduced into, or removed from, an experiment basket during any ATR outage. In the subsequent sections, the major test components that will comprise each test position in the ATR, namely the test rodlet, the capsule assembly, and the irradiation test assembly, will be described.

\subsection{Test Assembly}

The ATF-1 irradiation test assembly was based on an existing design that has been used since the late 1990s to test fueled rodlets in the ATR under conditions of temperature and power prototypic of LWRs. This design was originally developed to test mixed-oxide (MOX) fuels for the DOE Fissile Materials Disposition Program [4]. Although it continues to be referred to as the "MOX hardware" by ATR designers/experimenters, it has been used repeatedly for subsequent experiments to achieve irradiation conditions prototypic of an LWR.

\subsection{Capsule Assembly}

Each capsule will contain a single test rodlet (Figure 4). Since the fuels/cladding materials to be tested in the ATF-1 irradiation test series are to a large extent unproven, such that cladding breach during irradiation cannot be precluded in the safety analyses, secondary containment is required by ATR to ensure that fuel/fission products cannot be released into the primary coolant. A consequence of this design feature is that test rodlets must be hermetically sealed in order to contain fission gases, since polluting the gap between the capsule and rodlet with fission gases would increase the rodlet cladding and fuel temperatures above their design targets. 
Capsule length was fixed at 6.16 inches; however, the capsule can accommodate a shorter test rodlet as appropriate for some or all of the ATF fuel concepts to be tested. The radial dimensions of the capsule were also fixed, but the rodlet O.D. can be smaller and the I.D. can vary to meet test objectives, as determined from design analyses (Figure 5). A summary of capsule design parameters is given in Table 1.

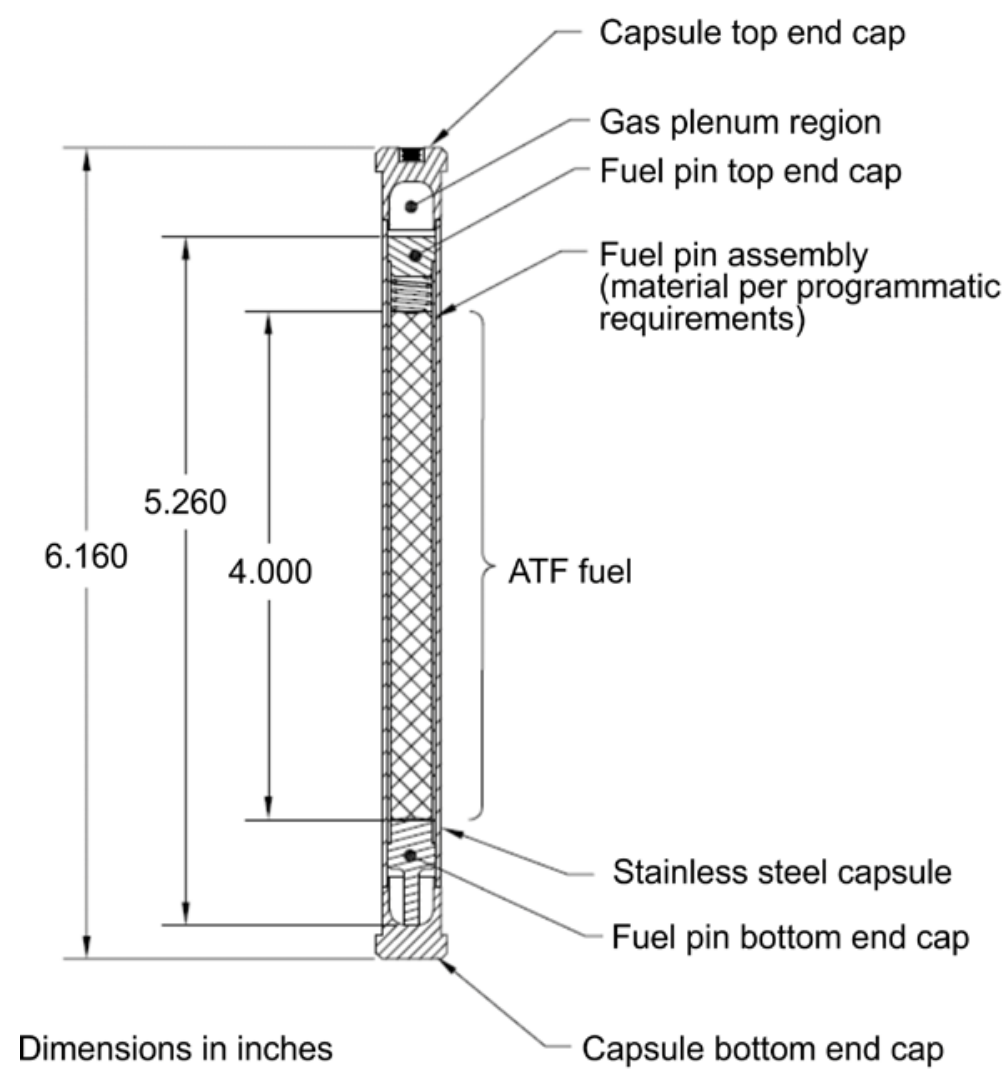

Figure 4. ATF-1 Test Capsule/Assembly (dimensions shown in inches). 


\section{ATF Test Capsule Design Nominal Dimensions}

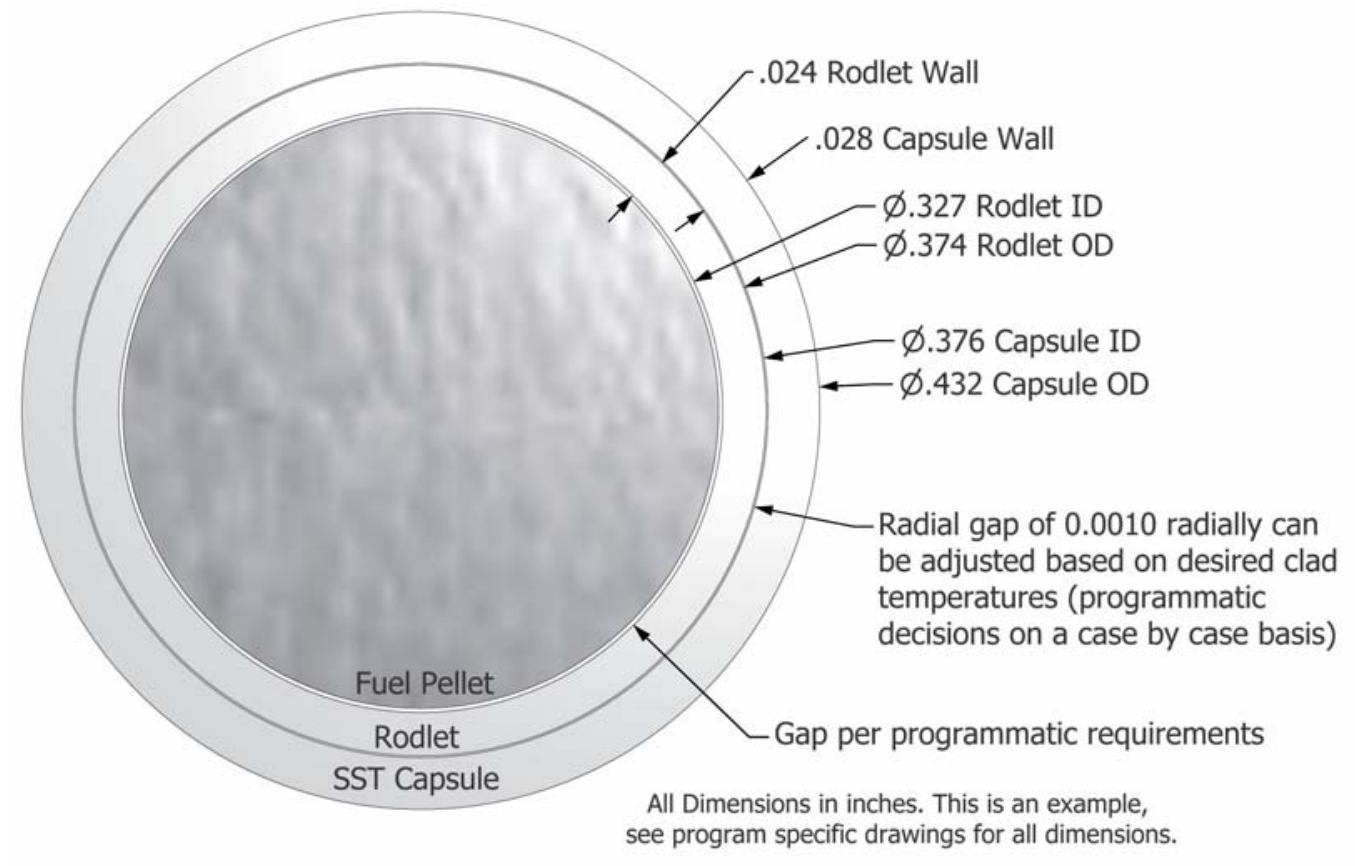

Figure 5. ATF-1 Capsule/Rodlet Cross Section (dimensions shown in inches).

Table 1. Capsule Design Data.

\begin{tabular}{|l|c|}
\hline Design Parameter & Value \\
\hline \hline Capsule Material & 316L SS \\
\hline Capsule O.D. & $1.10 \mathrm{~cm}(0.432$ in.) \\
\hline Capsule I.D. & $0.955 \mathrm{~cm}(0.376$ in.) \\
\hline Capsule Length & $15.646 \mathrm{~cm}(6.160$ in.) \\
\hline Capsule-Rodlet Gap & $\sim 0.003 \mathrm{~cm}(0.001$ in.) \\
\hline
\end{tabular}

If appropriate, capsules may be designed to accommodate non-fueled cladding materials, other fuel related materials, and/or empty "dummy" experiments (e.g., in the event that test specimens are not available for insertion during a particular cycle). Non-fuel materials may or may not need to be encapsulated inside a rodlet; it could be acceptable to encapsulate non-fuel materials directly inside a capsule, but this must be evaluated on a case-by-case basis.

\subsection{Test Rodlet}

The test rodlet is intended to represent a reduced length, pressurized water reactor (PWR) fuel rod, nominally prototypic in the radial dimension; the rodlet is shown along with the secondary stainless steel capsule in Figure 4 and Figure 5. For the ATF-1 irradiation test series, the test rodlet will (in general) contain fuel in the form of pellets or FCM compacts, a bonding agent, and a gas plenum, and it will be fabricated from various cladding materials and fuel forms outlined in the Test Matrix 
(Table 2 and Table 3). Table 2 identifies the test objectives for each concept team planned for FY 2014 design development. The test objectives include values for Peak Internal Cladding Temperature (PICT), Linear Heat Generation Rate (LHGR), Fuel Centerline (CL) Temperature, Burnup in Giga Watt days/Metric Ton (GWd/MT) in heavy metal, and ATR Effective Full Power Days. Table 3 identifies the test objectives for each concept team planned for FY 2015 design development including the number of rodlets

Table 2. FY 2014 ATF-1 Experiments.

\begin{tabular}{|c|c|c|c|c|c|c|c|c|}
\hline \multirow{3}{*}{$\begin{array}{c}\text { Lead } \\
\text { Organization }\end{array}$} & \multirow[b]{3}{*}{ Fuel } & \multirow[b]{3}{*}{ Cladding } & \multicolumn{6}{|c|}{ Target Test Conditions } \\
\hline & & & \multirow{2}{*}{$\begin{array}{l}\text { PICT } \\
\left({ }^{\circ} \mathrm{C}\right)\end{array}$} & \multirow{2}{*}{$\begin{array}{l}\text { LHGR } \\
(\mathrm{W} / \mathrm{cm})\end{array}$} & \multirow{2}{*}{$\begin{array}{c}\text { Fuel CL Temp } \\
\left({ }^{\circ} \mathrm{C}\right)\end{array}$} & \multicolumn{2}{|c|}{ Burnup } & \multirow[t]{2}{*}{$\begin{array}{l}\text { Number } \\
\text { Rodlets }\end{array}$} \\
\hline & & & & & & $(\mathrm{GWd} / \mathrm{MT})$ & (EFPD) & \\
\hline AREVA & $\begin{array}{l}\mathrm{UO}_{2} \\
\mathrm{UO}_{2}-\mathrm{Cr}_{2} \mathrm{O}_{3} \text { Doping } \\
\mathrm{UO}_{2} \text {-SiC Fibers } \\
\mathrm{UO}_{2} \text {-Diamond Particles }\end{array}$ & $\mathrm{Zr}-4$ & $300-420$ & $310-360$ & $\begin{array}{c}<1600 \\
\text { (700-1000 } \\
\text { calculated) }\end{array}$ & $\begin{array}{l}10 \\
50\end{array}$ & $\begin{array}{l}240 \\
700\end{array}$ & $\begin{array}{l}1 \\
1 \\
2 \\
2\end{array}$ \\
\hline Westinghouse & $\mathrm{U}_{3} \mathrm{Si}_{2}$ & Zirlo & $300-400$ & $270-470$ & $\begin{array}{c}480 \\
800 \\
1200\end{array}$ & $\begin{array}{l}20 \\
40 \\
70\end{array}$ & $\begin{array}{c}286 \\
571 \\
1000\end{array}$ & $\begin{array}{l}3 \\
2 \\
1\end{array}$ \\
\hline GE & $\mathrm{UO}_{2}$ & $\begin{array}{l}\text { Alloy } 33 \\
\text { APMT }\end{array}$ & $300-450$ & $315-330$ & $\begin{array}{c}1000-1200 \\
(1300 \\
\text { calculated) }\end{array}$ & $\begin{array}{l}20 \\
80\end{array}$ & $\begin{array}{c}325 \\
1300\end{array}$ & $\begin{array}{l}2 \\
2\end{array}$ \\
\hline ORNL & $\mathrm{UO}_{2}$ & $\mathrm{FeCrAl}$ & $300-400$ & $220-240$ & $\begin{array}{c}<1700 \\
(1200-1300 \\
\text { calculated })\end{array}$ & $\begin{array}{l}10 \\
30 \\
50\end{array}$ & $\begin{array}{c}240 \\
720 \\
1200\end{array}$ & $\begin{array}{l}1 \\
1 \\
1\end{array}$ \\
\hline
\end{tabular}


Table 3. FY 2015 - 2016 ATF-1 Planned Experiments.

\begin{tabular}{|c|c|c|c|c|c|c|c|c|}
\hline $\begin{array}{c}\text { Development } \\
\text { Lead } \\
\end{array}$ & Fuel & Cladding & Coating & \multicolumn{4}{|c|}{ Desired Irradiation Conditions: } & $\begin{array}{r}\text { Number } \\
\text { Rodlets }\end{array}$ \\
\hline ORNL & $\begin{array}{c}\text { FCM-UO }{ }_{2} \mathrm{UN} \\
(\mathrm{TBD})\end{array}$ & $\mathrm{Zr}-4$ & none & $180-280$ & $350-450 *$ & TBD & $10,30,50$ & 6, FY16 \\
\hline GE & $\begin{array}{c}\mathrm{UO}_{2} \\
(4.90 \% \mathrm{U}-235)\end{array}$ & Adv. Steel & none & $\sim 350$ & $300-400$ & $<1600$ & 20,80 & 2 \\
\hline EPRI / LANL & $\begin{array}{c}\mathrm{UO}_{2} \\
(4.95 \% \text { U-235) }\end{array}$ & Mo & $\begin{array}{l}\mathrm{Zr}-4 \text { or } \\
\text { FeCrAl } \\
\end{array}$ & $350-500$ & $300-360 *$ & $<1700$ & 25,50 & 8 \\
\hline U. Tennessee & $\mathrm{UO}_{2}$ & Zr-alloy & Ceramics & TBD & TBD & TBD & TBD & $\begin{array}{l}\text { TBD - } \\
\text { FY16 }\end{array}$ \\
\hline Georgia Tech & $\mathrm{U}_{3} \mathrm{Si}_{2}$ & $\mathrm{FeCrAl}$ & none & TBD & TBD & TBD & TBD & $\begin{array}{l}\text { TBD - } \\
\text { FY16 }\end{array}$ \\
\hline $\begin{array}{c}\text { Ceramic } \\
\text { Tubular } \\
\text { Products }\end{array}$ & $\begin{array}{c}\mathrm{UO}_{2} \\
(<5 \% \mathrm{U}-235)\end{array}$ & $\begin{array}{c}\text { SiC } \\
\text { TRIPLEX }\end{array}$ & none & TBD & $\begin{array}{l}300- \\
400 *\end{array}$ & $<1700$ & 20,50 & On hold \\
\hline LANL & $\begin{array}{c}3 \text { rodlets UN- } \\
15 \mathrm{v} \% / \mathrm{U}_{3} \mathrm{Si}_{5} \\
2 \text { rodlets } \\
\mathrm{UN} / \mathrm{U}_{3} \mathrm{Si}_{5} \\
(3-5 \% \mathrm{U}-235)\end{array}$ & FeCrAl & none & $\sim 240$ & $350 *$ & $<1650$ & $10,20,50$ & 5 \\
\hline
\end{tabular}

* Critical Parameter

The existing rodlet design accommodates a fuel column of approximately $10 \mathrm{~cm}$ (4 inches), although it should be possible to reduce this height if appropriate for certain ATF concepts. The fuel outer diameter (O.D.) and cladding inner diameter (I.D.) will be determined on a case-by-case basis for each ATF concept. However, the cladding O.D. is fixed at $0.968 \mathrm{~cm}(0.381$ inches) in order to establish the gas gap between the rodlet cladding O.D. and the capsule I.D. determined by thermal analyses to be necessary to elevate the rodlet cladding temperature to its target value. A summary of the test rodlet design data is given in Table 4 . 
Table 4. Rodlet Design Data.

\begin{tabular}{|l|c|}
\hline Design Parameter & Value \\
\hline \hline Cladding Material & Zircaloy/other \\
\hline Cladding O.D. & $0.968 \mathrm{~cm}(0.381$ in.) \\
\hline Cladding I.D. & TBD \\
\hline Rodlet Fill Gas & Helium \\
\hline Fuel Type & $\mathrm{UO}_{2} / \mathrm{ATF}$ \\
\hline Fuel O.D. & $\mathrm{TBD}$ \\
\hline Fuel Column Height & $\leq 10.1 \mathrm{~cm}(4.0$ in. $)$ \\
\hline
\end{tabular}

\subsection{Basket Assembly}

The experiment basket functions as an interface between the capsule assembly and the ATR irradiation position. The preliminary basket design is a 3 channel aluminum basket. As seen in Figure 6, the basket design incorporates space for flux monitors (if desired) to be measured during PIE. It also provides for a fixed azimuthal orientation relative to ATR core center. Each basket can hold up to seven $15.65 \mathrm{~cm}$ (6 inch) capsules per channel for a maximum of 21 capsules per basket (Figure 7).

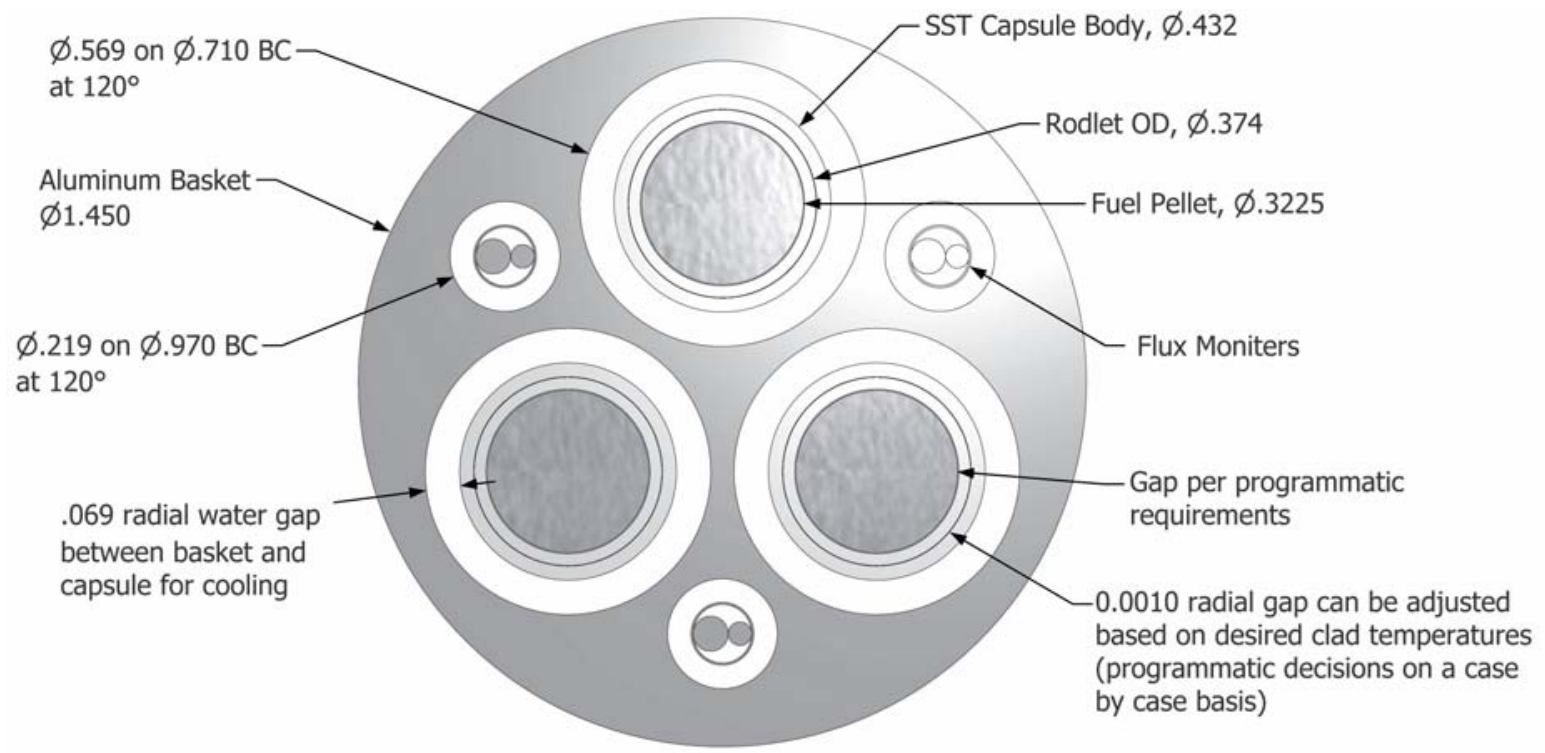

All Dimensions in inches. This is an example, see program specific drawings for all dimensions.

Figure 6. Cross-section of ATF-1 basket assembly (dimensions provided in inches). 


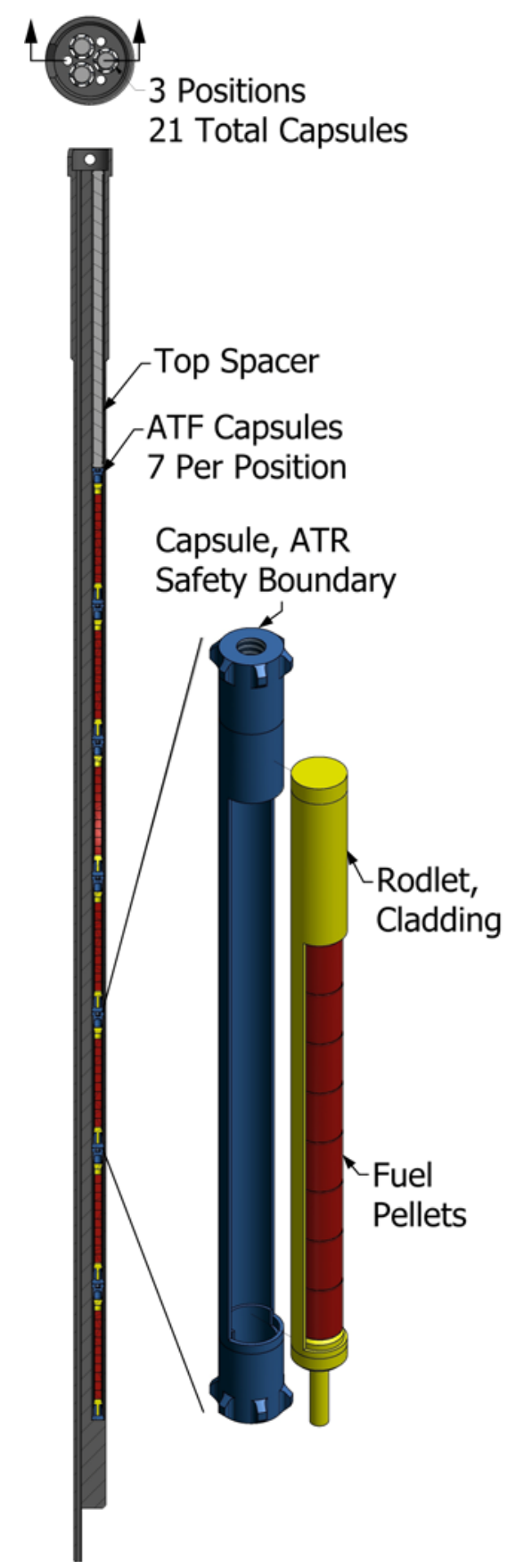

Figure 7. ATF basket assembly showing stack of 7 capsules in one of the three channels.

However, the ATR axial flux profile (Figure 8) shows that flux drops dramatically at the top and bottom of the basket; therefore, most basket configurations for ATF-1 testing will include a combination of fueled capsules and dummy capsules configured in the basket to reach optimum irradiation conditions to meet test objectives. 


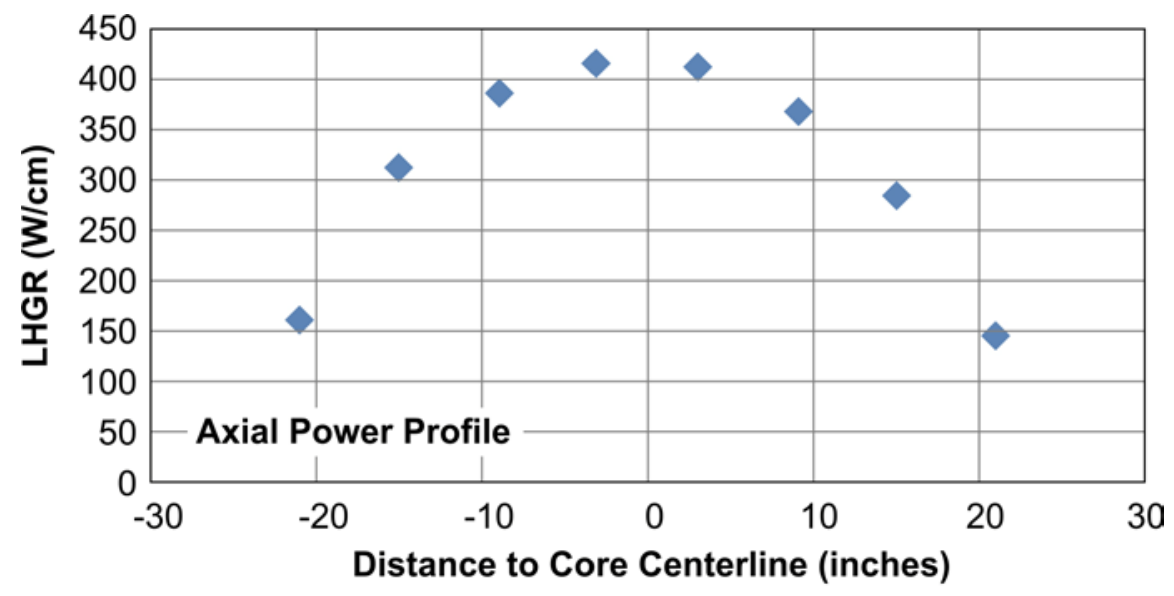

Figure 8. Calculated LHGR as a function of axial position in the test train (for $5 \%$ enriched $\mathrm{UO}_{2}$ ).

\subsection{Irradiation Test Conditions}

The ATF basket assembly can be accommodated in either the small-I or large-B test positions in the ATR reflector. Design analyses identified the small-I positions shown in Figure 9 as the optimum ATR positions for LWR prototypic flux conditions. These four small-I positions are designated as I-21 in the northeast (NE) corner, I-22 in the southeast (SE) corner, I-23 in the southwest (SW) corner, and I-24 in the northwest (NW) corner of the ATR; these positions have nominal (unperturbed) flux levels of $8.4 \times 10^{13} \mathrm{n} / \mathrm{cm}^{2}$-sec (thermal) and $3.2 \times 10^{12} \mathrm{n} / \mathrm{cm}^{2}$-sec (fast), although these levels vary depending on actual lobe operating powers during any particular cycle. At least 2 (and perhaps all 4) of these small-I positions were available for use in the ATF-1 irradiation test series beginning in FY-2014.

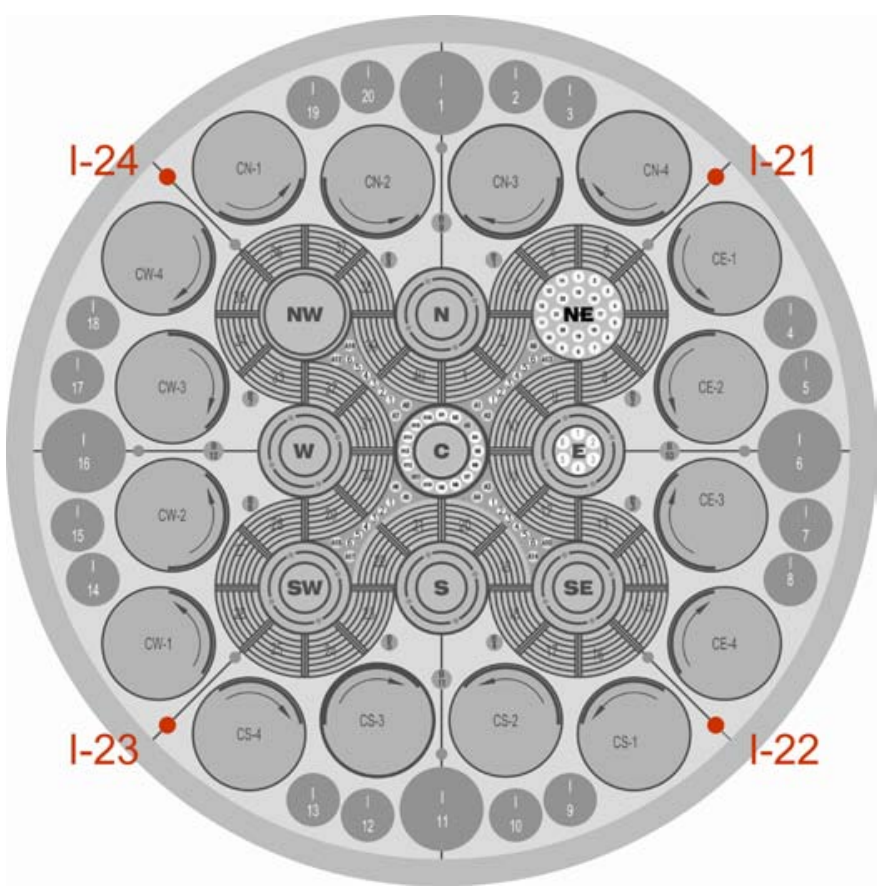

Figure 9. Cross-section of ATR core highlighting locations of the 4 small-I positions. 


\section{METHODOLOGY}

In preparation for ATR experiment testing, test specimen conceptual designs are created in collaboration with the ATF concept development team leads as identified in Table 2 and Table 3. Once a conceptual design is approved by the design team and the development team leads, an iterative series of design analyses are performed as identified in Figure 3 to identify the final test specimen nominal dimensions, nominal lobe power, and nominal fuel enrichment. Once the analysis results are accepted and approved, the final drawings are released which include dimensional tolerances primarily based on machining capabilities and sensitive analyses performed to identify the acceptable range fuel, rodlet, and capsule gap sizes. Details of the design and analysis process are described in the following sections.

\subsection{Design Process}

This section describes processes required for all ATR irradiation testing experiments including the ATF experiments. One of the initial challenges that must be addressed during the design phase of test development was the experiment geometry and compatibility with the reactor test facility. Where possible, fuel cladding systems intended for in-pile testing should be designed to take advantage of existing analyses, designs, and structures developed for previous experiments at the selected test reactor. Limiting the design to a previously accepted configuration reduces the cost and allows a faster start to the irradiation testing. However, the design engineer and analysts must examine the previous fabrication and analysis techniques used and assumptions made in the design, analysis, and fabrication process. In many instances, an existing design may have been fabricated at a different facility using specialized equipment. Assumptions applied and accepted in the original design analysis may no longer be valid or accepted. New requirements may be enforced in the analyses. All these factors should be fully explored before making a decision to adopt an existing or previously used design.

Test reactors often require that all materials in contact with the reactor primary coolant be "known," denoting that material compositions must be certified and traceability of materials must be confirmed. In addition, incompatible constituents may be restricted and/or only allowed under special conditions. Quality control challenges exist for procurement of nontraditional/innovative materials primarily due to unavailability of materials or components through NQA-1 compliant vendors. To allow procurement from non-NQA-1 vendors, special processes such as vendor assessments, receipt inspections, and independent material analyses (to verify vendor certifications) must be implemented.

Design considerations should include methods for chemically and/or mechanically bonding layered clad materials together, hermetically sealing the rodlet, material weld requirements, especially for innovative clad materials, feasibility of fabrication and assembly, special fabrication and assembly equipment needed, special inspection equipment needed, and ease of disassembly in a hot cell after irradiation for post-irradiation examination (PIE). Modeling simulations should be performed to evaluate design performance with in-reactor test conditions. Full mock-up assemblies should be fabricated to verify that these steps can be achieved and to determine if new procedures and/or equipment are needed to successfully fabricate, assemble, and inspect the experiment assembly.

\subsection{Design Analysis Process}

ATR experiments must demonstrate that they meet specified thermal safety requirements. From a thermal/hydraulic standpoint, this amounts to ensuring that the experiment will not exceed the limiting case (i.e., flow excursion) in which critical heat flux or coolant saturation temperatures cannot be exceeded, will not melt, and will not cause adverse reactivity effects during postulated reactivity transients. Coolant provided to the experiment must be sufficient to meet these limits, but not high enough to reduce flow to the reactor driver elements. The outer capsule is the safety pressure boundary component of the experiment and must be evaluated to verify that the capsule design will meet ATR safety requirements. The capsule is designed and analyzed to meet the intent of the ASME Section III Class 1 code requirements. Irradiation performance of ASME Section III Class 1 materials is well documented and, therefore, is not included in the sensitivity analyses outlined in this paper. The inner test article fuel rodlet is not identified as a safety component since the rodlet is assumed to breach in the safety analyses (this is not desirable programmatically, but offers a conservative analysis) to verify that fission gas release to the capsule during irradiation would not breach the pressure boundary capsule. 
Finite element models of the experiment are used to evaluate experiment performance during irradiation testing in the ATR. Material and structural properties used as model input were based on beginning of life (BOL) data for the innovative test components since irradiated material property data are not available. Temperature results from these analyses feed into structural analyses that evaluate experiment performance based on thermal stress loads. The results of the analyses are provided as part of the programmatic risk assessment prior to insertion to determine the confidence level for meeting test objectives.

\subsubsection{ATF-1 Design Analyses}

An ABAQUS finite element model of a basket with 21 capsules was generated to evaluate the best estimate temperatures during assumed reactor conditions. The model includes a ring of beryllium representative of the reflector surrounding the Small I position. The fuel pellets, insulator pellets, and rodlets are included in the model.

The gap between the capsule and the rodlet will change due to thermal expansion as the materials heat up. This effect was analyzed using a 2D ABAQUS model that includes both temperature and thermal expansion effects. This model was used to compute the dimensions of the rodlet and capsule (and by extension, the gap between them) at several temperatures. The change in the gap size was incorporated into the full 3D thermal analysis by modifying the heat transfer coefficient between the rodlet and capsule surfaces. The change in the heat transfer corresponding to gap geometry at the analyzed temperatures was incorporated into the 3D model. A check was performed to determine that the gap size was based on a temperature that was consistent with the final analysis results.

ABAQUS is a finite-element code that can evaluate the heat conduction through materials in three dimensions, internal heat generation within the materials, and heat transfer properties between the fuel rodlet and the capsule fill gas. ABAQUS finiteelement models are constructed to evaluate the thermal performance of the experiment while inserted in the reactor. The ABAQUS model of the ATF experiment was developed to match the basket, capsules, and rodlets as designed and documented in experiment design drawings. The parts were all modeled using ABAQUS solid elements.

Convective water flow through the model accounts for the transport of the gamma heat generated in the experiment to the reactor primary coolant. Heating in the experiment was from any included fuel and gamma absorption by the experiment materials. The BOL heating rates calculated using MCNP (a general purpose Monte Carlo N-Particle transport code), for fuel and experiment materials are provided as input to the ABAQUS model. These base heating rates are increased by factors to account for maximum reactor power operations and instrumentation uncertainties. Structural evaluation begins with temperatures from the thermal analysis as shown in the flow diagram (Figure 3). In addition, mechanical properties of the cladding material such as modulus of elasticity, Poisson's ratio, coefficient of thermal expansion, yield strength, and tensile strength are also required. Note that the variation of the mechanical properties with temperature was important to provide reasonably accurate calculations.

Loads and boundary conditions are the next step in the structural evaluation. Loads come from two sources. First, the temperature gradient through the material induces thermal stresses. Second, internal and external pressures induce mechanical stresses. Boundary conditions must properly represent the restrictions on material movement.

Typically, a structural evaluation is applied to calculate cladding displacements and stresses. For simple cladding materials and geometries, closed form solutions may be available. Otherwise, a finite element model would be constructed. The cladding must be evaluated for excessive deflections and failure. If the cladding deflects enough that it contacts the capsule inside wall, the experiment cladding may not reach or maintain the desired temperature. Cladding failure may be classified as ductile or brittle depending on the material. For a ductile material, the burst strength can be estimated from the stresses. For a brittle material, the fracture strength will need to be determined. If the cladding consists of two different materials that are bonded together, the nature of the bond between the materials needs to be investigated. For example, differences in coefficients of thermal expansion may produce significant stresses at the bond interface. Also, the strength of the bond itself may need to be characterized. The bond strength may be affected by temperature and stress such that, under certain conditions, the bond will fail. 
If the structural criteria cannot be met, then changes to the thermal conditions and/or neutronics conditions will be needed, as shown in the flow diagram (Figure 3). The cladding thickness may need to be modified as well. These analysis iterations can be time consuming and result in changes to the cladding thickness.

\subsection{Sensitivity Analyses}

The ABAQUS model requires detailed information about thermal conductivities, emissivities, and heat transfer coefficients in order to generate accurate results. The innovative cladding designs and materials being considered have often not been studied in enough depth to have generated the required data. Often, the thermal conductivity and other parameters are somewhat dependent on the manufacturing process and type of materials used. In order to adequately evaluate experiment temperatures and performance under irradiation conditions, it was necessary to understand the sensitivity of property uncertainties on the temperature, pressure, and stress calculations. A sensitivity analysis was initially performed using the ABAQUS model which was beneficial in identifying the dimensional and lobe power tolerances for the experiment. However, it was recognized during the experiment design phase that several material and structural properties of the cladding and fuels being tested were virtually unknown for the innovative materials under irradiation conditions. Although a stack-up of all parameter uncertainties could result in a higher degree of design analysis uncertainties, the process is time consuming and costly, and the results will have a high degree of uncertainty due to lack of irradiation testing data available. Therefore, INL analysts performed a Taylor Series sensitivity analysis for propagation of uncertainties in temperature and pressure calculations to compare the relative degree of parameter uncertainties of the calculations. The results were used to identify the most critical parameters for experiment design and risk assessment.

\subsubsection{Taylor Series Analysis}

In general, the important experiment parameters for predicting fuel rodlet performance, such as cladding temperature, are calculated from an equation that will have many variables [Eq. 1]. These variables usually include geometric properties such as inside and outside diameter, as well as material properties, such as thermal conductivity and convection heat transfer coefficients. These variables are selected randomly around a mean and standard deviation associated with each one of them. The calculated temperature will also be a random variable. Hence, the expected temperature (mean value) and the uncertainty (standard deviation) are desired. Suppose the temperature can be expressed as a function of $\mathrm{n}$ variables (x);

$$
T=T\left(x_{1}, x_{2}, x_{3}, \ldots \ldots, x_{n}\right) \quad \text { Eq. } 1
$$

The average value of the temperature can be estimated by evaluating the equation at the averages of all the variables [Eq. 2];

$$
T_{\mu}=T\left(x_{1 \mu}, x_{2 \mu}, x_{3 \mu}, \ldots \ldots, x_{n \mu}\right) \quad \text { Eq. } 2
$$

The standard deviation of the temperature can be estimated using Eq. 3

$$
T_{\sigma}=\sqrt{\left[\sum_{i=1}^{n}\left(\frac{\partial T}{\partial x_{i}}\right)_{\mu}^{2}\left(x_{i \sigma}\right)^{2}\right]} \quad \text { Eq. } 3
$$

The first term in the summation in Equation 3 is the partial derivative with respect to each variable evaluated at the average value of that variable. The second term is the variance of each variable. Equations 2 and 3 are often called the Taylor series method for propagation of uncertainties. . The following assumptions and restrictions apply:

- Equation 1 is assumed to be continuous and has continuous derivatives in the domain of interest [5]

- Equations 2 and 3 are approximate because the Taylor series was truncated after the linear term [6]

- Equations 2 and 3 provide estimates for the mean and standard deviation of the temperature but the statistical distribution of the temperature is unknown [6].

- Correlation effects are not included. In general, correlation effects can be separated into systematic error and random error. If all the variables are independent, systematic error correlation effects are negligible. If the random errors are 
uncorrelated, then the random error correlation effects can be ignored. For this analysis, no correlation effects were considered or investigated [5].

Coleman and Steele define two factors that are helpful in evaluating the effect of each variable on the temperature. The first is the uncertainty magnification factor (UMF) defined as [Eq. 4;

$$
U M F_{i}=\frac{x_{i}}{T_{\mu}} \frac{\partial T}{\partial x_{i}} \quad E q .4
$$

The UMF for a given variable indicates the influence of the uncertainty in that variable on the square of the uncertainty in the result. A UMF value greater than 1 indicates that the influence of the variable uncertainty is magnified as it propagates through the temperature equation. A UMF value of less than 1 indicates that the influence of the variable uncertainty is diminished as it propagates through the temperature equation. The second factor is the uncertainty percentage contribution (UPC) defined as [Eq. 5];

$$
U P C_{i}=\frac{\left(\frac{\partial T}{\partial x_{i}}\right)^{2} x_{i \sigma}^{2}}{T_{\sigma}^{2}} \cdot 100 \quad \text { Eq.5 }
$$

The UPC for a given variable gives the percentage contribution of the uncertainty in that variable to the squared uncertainty in the temperature result.

To demonstrate the application of the Taylor Series Method to determine the experiment design critical parameters, an APMT advanced steel clad rodlet was selected as an example and sensitivity analyses results are presented in this paper.

The APMT rodlet parameters calculated in the Taylor Series Sensitivity Analysis included:

- allowable rodlet stress (structural calculations),

- $\quad$ peak internal cladding temperature (thermal calculations), and

- maximum allowable rodlet pressure during irradiation testing

\section{RESULTS}

A sensitivity analysis was initially performed using the ABAQUS model which was beneficial in identifying the dimensional and lobe power tolerances for the experiment. However, it was recognized during the experiment design phase that several material and structural properties of the cladding and fuels being tested were virtually unknown for the innovative materials under irradiation conditions. Although a stack-up of all parameter uncertainties could result in a higher degree of design analysis uncertainties, the process is time consuming and costly, and the results will have a high degree of uncertainty due to lack of irradiation testing data available. Therefore, INL analysts performed a Taylor Series sensitivity analysis for propagation of uncertainties in temperature and pressure calculations to compare the relative degree of parameter uncertainties of the calculations. The results were used to identify the most critical parameters for experiment design and risk assessment.

\subsection{ABAQUS Sensitivity Analyses Results}

A sensitivity analysis was performed using ABAQUS during the experiment design phase to determine the effect that changes to the gas gap between the rodlet and capsule and the reactor power level during irradiation may have on the experiment. The temperatures calculated using the nominal rodlet and capsule dimensions and nominal ATR lobe power (19 MW) for 2 different advanced steels (Alloy-33 and APMT) are shown in Table 5. The temperatures resulting from a 0.001 inch increase in the gap size between the capsule and cladding (i.e. rodlet tube) are shown in Table 6. A decrease of 0.001 inch in the gap size will produce the temperature results shown in Table 7. The results indicate that a 0.001 inch change in the 
gas gap between the capsule and the cladding will change the cladding volume weighted average temperature by about \pm $20 \%$. The maximum and minimum fuel temperatures are also impacted by about $\pm 10 \%$.

Table 5. Temperature Results Using Nominal Rodlet and Capsule Dimension.

\begin{tabular}{|c|c|c|c|c|}
\hline & $\begin{array}{c}\text { Pellet Max } \\
\text { Temp. }\left({ }^{\mathbf{0}} \mathrm{C}\right)\end{array}$ & $\begin{array}{c}\text { Pellet Min } \\
\text { Temp. }\left({ }^{\mathbf{0}} \mathrm{C}\right)\end{array}$ & $\begin{array}{c}\text { Rodlet Max } \\
\text { Temp. }\left({ }^{\mathbf{0}} \mathbf{C}\right)\end{array}$ & $\begin{array}{c}\text { Rodlet Vol } \\
\text { Wt. Avg } \\
\text { Temp. }\left({ }^{\mathbf{0}} \mathbf{C}\right)\end{array}$ \\
\hline \hline $\begin{array}{c}\text { Channel 3 } \\
\text { Position 2 } \\
\text { (APMT) }\end{array}$ & 1516.3 & 348.7 & 381.5 & 349.4 \\
\hline $\begin{array}{c}\text { Channel 3 } \\
\text { Position 6 } \\
\text { (Alloy-33) }\end{array}$ & 1504.4 & 329.6 & 353.6 & 323.7 \\
\hline $\begin{array}{c}\text { Channel 1 } \\
\text { Position 2 } \\
\text { (APMT) }\end{array}$ & 1485.4 & 346.0 & 376.6 & 344.9 \\
\hline $\begin{array}{c}\text { Channel 1 } \\
\text { Position 6 } \\
(\text { Alloy-33) }\end{array}$ & 1501.2 & 328.2 & 353.1 & 323.2 \\
\hline
\end{tabular}

Table 6. Temperature Results From Gap Sensitivity (+0.001 inches).

\begin{tabular}{|c|c|c|c|c|}
\hline & $\begin{array}{c}\text { Pellet Max } \\
\text { Temp. }\left({ }^{\circ} \mathrm{C}\right)\end{array}$ & $\begin{array}{c}\text { Pellet Min } \\
\text { Temp. }\left({ }^{\circ} \mathrm{C}\right)\end{array}$ & $\begin{array}{c}\text { Rodlet Max } \\
\text { Temp. }\left({ }^{\circ} \mathrm{C}\right)\end{array}$ & $\begin{array}{c}\text { Rodlet Vol } \\
\text { Wt. Avg } \\
\text { Temp. }\left({ }^{\circ} \mathrm{C}\right)\end{array}$ \\
\hline \hline $\begin{array}{c}\text { Channel 3 } \\
\text { Position 2 } \\
\text { (APMT) }\end{array}$ & 1601.3 & 378.9 & 451.6 & 418.2 \\
\hline $\begin{array}{c}\text { Channel 3 } \\
\text { Position 6 } \\
\text { (Alloy-33) }\end{array}$ & 1581.3 & 355.5 & 416.6 & 385.1 \\
\hline $\begin{array}{c}\text { Channel 1 } \\
\text { Position 2 } \\
\text { (APMT) }\end{array}$ & 1570.1 & 375.9 & 445.7 & 412.7 \\
\hline $\begin{array}{c}\text { Channel 1 } \\
\text { Position 6 } \\
\text { (Alloy-33) }\end{array}$ & 1578.1 & 354.1 & 415.9 & 384.6 \\
\hline
\end{tabular}


Table 7. Temperature Results From Gap Sensitivity (-0.001 inches).

\begin{tabular}{|c|c|c|c|c|}
\hline & $\begin{array}{c}\text { Pellet Max } \\
\text { Temp. }\left({ }^{\circ} \mathrm{C}\right)\end{array}$ & $\begin{array}{c}\text { Pellet Min } \\
\text { Temp. }\left({ }^{\circ} \mathrm{C}\right)\end{array}$ & $\begin{array}{c}\text { Rodlet Max } \\
\text { Temp. }\left({ }^{\circ} \mathrm{C}\right)\end{array}$ & $\begin{array}{c}\text { Rodlet Vol } \\
\text { Wt. Avg } \\
\text { Temp. }\left({ }^{\circ} \mathrm{C}\right)\end{array}$ \\
\hline $\begin{array}{c}\text { Channel 3 } \\
\text { Position 2 } \\
\text { (APMT) }\end{array}$ & 1422.1 & 317.1 & 305.5 & 274.5 \\
\hline $\begin{array}{c}\text { Channel 3 } \\
\text { Position 6 } \\
\text { (Alloy-33) }\end{array}$ & 1419.8 & 302.6 & 285.6 & 257.1 \\
\hline $\begin{array}{c}\text { Channel 1 } \\
\text { Position 2 } \\
\text { (APMT) }\end{array}$ & 1391.6 & 314.6 & 301.6 & 271.0 \\
\hline $\begin{array}{c}\text { Channel 1 } \\
\text { Position 6 } \\
\text { (Alloy-33) }\end{array}$ & 1416.6 & 301.3 & 285.2 & 256.8 \\
\hline
\end{tabular}

The temperatures corresponding to a power increase of 1 MW (19 MW to 20 MW) are shown in Table 8. The same results are shown in Table 9 for a power decrease of $1 \mathrm{MW}$ (19 MW to $18 \mathrm{MW}$ ). The results indicate that a $1 \mathrm{MW}$ change in lobe power will change the rodlet weighted average temperature by about $\pm 4 \%$ and maximum and minimum fuel temperatures by about $\pm 6 \%$.

Table 8. Temperature Results From Power Sensitivity (+1MW).

\begin{tabular}{|c|c|c|c|c|}
\hline & $\begin{array}{c}\text { Pellet Max } \\
\text { Temp. }\left({ }^{\circ} \mathrm{C}\right)\end{array}$ & $\begin{array}{c}\text { Pellet Min } \\
\text { Temp. }\left({ }^{\circ} \mathrm{C}\right)\end{array}$ & $\begin{array}{c}\text { Rodlet Max } \\
\text { Temp. }\left({ }^{\circ} \mathrm{C}\right)\end{array}$ & $\begin{array}{c}\text { Rodlet Vol } \\
\text { Wt. Avg } \\
\text { Temp. }\left({ }^{\circ} \mathrm{C}\right)\end{array}$ \\
\hline $\begin{array}{c}\text { Channel 3 } \\
\text { Position 2 } \\
\text { (APMT) }\end{array}$ & 1604.7 & 361.1 & 395.7 & 362.4 \\
\hline $\begin{array}{c}\text { Channel 3 } \\
\text { Position 6 } \\
\text { (Alloy-33) }\end{array}$ & 1593.3 & 341.3 & 366.8 & 335.7 \\
\hline $\begin{array}{c}\text { Channel 1 } \\
\text { Position 2 } \\
\text { (APMT) }\end{array}$ & 1572.4 & 358.3 & 390.4 & 357.7 \\
\hline $\begin{array}{c}\text { Channel 1 } \\
\text { Position 6 } \\
\text { (Alloy-33) }\end{array}$ & 1589.9 & 339.8 & 366.3 & 335.2 \\
\hline
\end{tabular}


Table 9. Temperature Results From Power Sensitivity (-1MW).

\begin{tabular}{|c|c|c|c|c|}
\hline & $\begin{array}{c}\text { Pellet Max } \\
\text { Temp. }\left({ }^{\circ} \mathrm{C}\right)\end{array}$ & $\begin{array}{c}\text { Pellet Min } \\
\text { Temp. }\left({ }^{\circ} \mathrm{C}\right)\end{array}$ & $\begin{array}{c}\text { Rodlet Max } \\
\text { Temp. }\left({ }^{\circ} \mathrm{C}\right)\end{array}$ & $\begin{array}{c}\text { Rodlet Vol } \\
\text { Wt. Avg } \\
\text { Temp. }\left({ }^{\circ} \mathrm{C}\right)\end{array}$ \\
\hline $\begin{array}{c}\text { Channel 3 } \\
\text { Position 2 } \\
\text { (APMT) }\end{array}$ & 1427.1 & 336.1 & 367.2 & 336.3 \\
\hline $\begin{array}{c}\text { Channel 3 } \\
\text { Position 6 } \\
\text { (Alloy-33) }\end{array}$ & 1414.3 & 317.7 & 340.2 & 311.5 \\
\hline $\begin{array}{c}\text { Channel 1 } \\
\text { Position 2 } \\
\text { (APMT) }\end{array}$ & 1397.2 & 333.5 & 362.4 & 331.9 \\
\hline $\begin{array}{c}\text { Channel 1 } \\
\text { Position 6 } \\
\text { (Alloy-33) }\end{array}$ & 1411.2 & 316.4 & 339.7 & 311.1 \\
\hline
\end{tabular}

\subsection{Taylor Series Approach Sensitivity Analysis Results}

Neutronic, thermal, and structural calculations were performed to calculate UPC and UMF for a series of variables that contribute uncertainties to the rodlet parameter calculations. The results are presented for each set of calculations using the Taylor Series Approach.

\subsubsection{Neutronics Calculations}

The pressure of the gas inside of a rodlet can be estimated using the ideal gas equation $P V=n R T$, where $P$ is the gas pressure, $\mathrm{V}$ is the volume of the gas, $\mathrm{n}$ is the number of moles of gas, which is a function of power, time, and fission gas release rate. Time is assumed to be a well-known value with little to no variation and is thus neglected. The volume of the gas can be estimated as the volume between a cylinder inside of a cylinder. Thus, the pressure inside of the rodlet can be expressed as [Eq. 6]:

$$
\boldsymbol{P}=\boldsymbol{P}(\boldsymbol{r} \mathbf{1}, \boldsymbol{r} 2, \boldsymbol{h 1}, \boldsymbol{h 2}, \boldsymbol{F 1}, \boldsymbol{T g}) \emptyset \quad \text { Eq. } 6
$$

Where:

$$
\begin{aligned}
& \mathrm{P}=\text { Rodlet internal pressure } \\
& \mathrm{r} 1=\text { Fuel radius } \\
& \mathrm{r} 2=\text { Rodlet inside radius } \\
& \mathrm{h} 1=\text { Fuel height } \\
& \mathrm{h} 2=\text { Rodlet height } \\
& \mathrm{F} 1=\text { Fission gas release rate } \\
& \phi=\text { Average power of the fuel } \\
& \mathrm{Tg}=\text { Temperature of the gas }
\end{aligned}
$$

For this analysis, the average and coefficients of variation (CV) for each of the variables are shown in Table 10. Tight control of the tolerances on the rodlet dimensions allow for very small CV values of $r 1, r 1, h 1, h 2$. Uncertainty in reactor power is the primary driver of the larger $\mathrm{CV}$ value for power and temperature whereas experimental unknown contributes to the largest $\mathrm{CV}$ value in the fission gas release rate. 
Table 10. Average and CV values used in the neutronics sensitivity analysis.

\begin{tabular}{|l|l|l|}
\hline \multicolumn{1}{|c|}{ Variable } & Average, $\boldsymbol{\mu}$ & $\mathbf{C V}, \boldsymbol{\sigma} / \boldsymbol{\mu}$ \\
\hline Fuel radius (r1) & $4.10 \mathrm{~mm}$ & 0.005 \\
\hline Rodlet inside radius (r2) & $4.15 \mathrm{~mm}$ & 0.003 \\
\hline Fuel height (h1) & $102 \mathrm{~mm}$ & 0.005 \\
\hline Rodlet height (h2) & $108 \mathrm{~mm}$ & 0.005 \\
\hline Fission gas release rate (F1) & 0.25 & 0.25 \\
\hline $\begin{array}{l}\text { Average power of the fuel } \\
(\phi)\end{array}$ & $350 \mathrm{~W} / \mathrm{cm}$ & 0.1 \\
\hline Temperature of the gas (Tg) & $627 \mathrm{~K}$ & 0.1 \\
\hline
\end{tabular}

Note that there are a number of assumed constants which are neglected in this analysis such as time, energy per fission, and fission gas molecules per fission as their effect on the uncertainty of the calculation are either negligible or extremely hard to quantify. After factoring in these constants the average, standard deviation, and resulting coefficient of variation of the pressure becomes [Eq. 7, Eq. 8, and Eq. 9]:

$$
\begin{array}{lc}
\boldsymbol{P}_{\boldsymbol{\mu}}=\mathbf{1 2 . 2} \mathbf{M P a} & \text { Eq.7 } \\
\boldsymbol{P}_{\boldsymbol{\sigma}}=\mathbf{4 . 0 3} \mathbf{M P a} & \text { Eq. } 8 \\
\boldsymbol{P}_{\boldsymbol{C V}}=\frac{\boldsymbol{P}_{\sigma}}{\boldsymbol{P}_{\boldsymbol{\mu}}}=\mathbf{0 . 3 2 8} & \text { Eq. } 9
\end{array}
$$

Using Eq. 4, the uncertainty percentage contribution, UPC, for each variable can be calculated. These values are shown in Figure 10. As can be seen from the figure, the fission gas release rate (F1) was the dominant contributor to the UPC. The UMF was calculated using Eq. 5 with the results shown in Figure 11. The time duration in the calculation was 1200 days. As can be seen from the figure, the dimensional factors are the dominant variables for UMF. Tight dimensional tolerance significantly reduces the contribution of the dimensional factors to the overall pressure uncertainty. This makes the fission gas release rate by far the largest overall contributor to pressure calculation uncertainty. Alone it accounted for over $50 \%$ of the total variation in the rodlet internal pressure. 


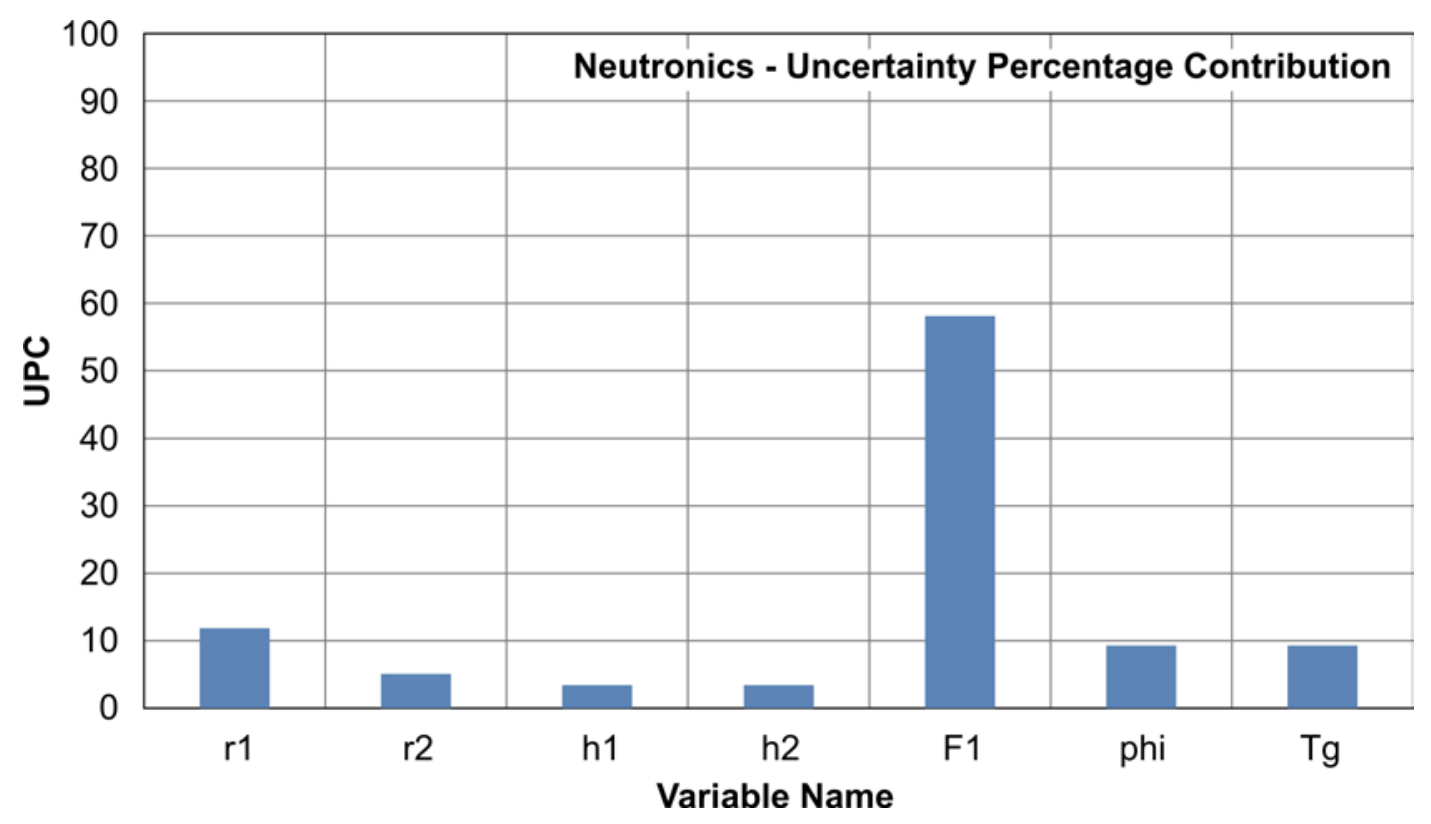

Figure 10: UPC values for neutronics analysis.

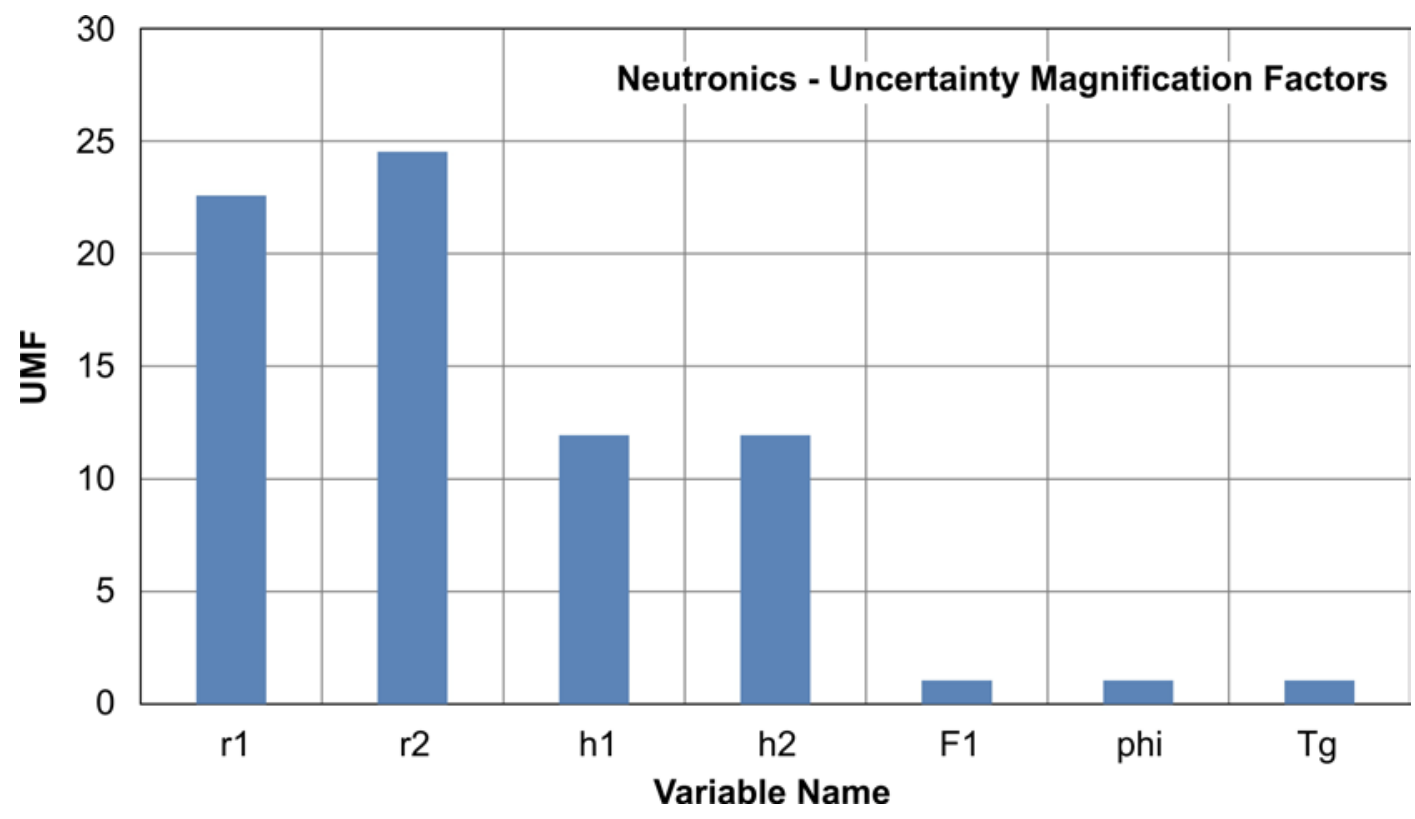

Figure 11: UMF values for neutronics analysis.

\subsubsection{Thermal Calculations}

The inner wall temperature of the rodlet can be modeled as a function of the following variables [Eq. 10]:

$$
T_{\text {in }}=T_{\text {in }}\left(T_{\text {cool }}, q, r 1, r 2, r 3, r 4, r 5, k A, k B, k C, k D, k E, h\right)
$$

Where:

$\mathrm{T}_{\mathrm{in}}=$ Temperature at inside rodlet surface 
$\mathrm{T}_{\text {cool }}=$ Temperature of coolant outside capsule

$\mathrm{q}=$ Fuel pellet (inside rodlet) LHGR

r2 $=$ Rodlet inside radius

r3 = Rodlet outside radius

$\mathrm{r} 4$ = Capsule inside radius

r5 = Capsule outside radius

$\mathrm{kC}=$ Rodlet thermal conductivity

$k D=$ Helium gap thermal conductivity (rodlet to capsule)

$k E=$ Capsule thermal conductivity

$h=$ Heat transfer coefficient from capsule to coolant

The temperature computed by the function in Equation 10 shows no specific dependence on the radius of the fuel pellet. This carries the assumption that the heating rate in the fuel $(q)$ is not dependent on the fuel pellet dimensions. In practice, this can only be accomplished if the enrichment of the fuel or the reactor power is flexible. If both of these parameters are determined by outside requirements, then the LHGR will not be independent of the pellet dimensions, and they should be included as parameters that affect the rodlet temperatures.

For this analysis, the average and coefficients of variation (CV) for each of the variables are shown in Table 11. Note that thermal expansion will affect the dimensions of the capsule and the rodlet. This analysis does not include the thermal expansion effects, which require an iterative solution that was not applicable to this method. Using Eq. 2 and Eq. 3, the resulting average, standard deviation, and coefficient of variation for the rodlet inside temperatures are:

$$
\begin{aligned}
& \boldsymbol{T}_{\text {in }, \boldsymbol{\mu}}=554.48 \mathrm{~K} \quad \text { Eq. } 11 \\
& \boldsymbol{T}_{\boldsymbol{i n}, \boldsymbol{\sigma}}=123 \mathrm{~K} \quad \text { Eq. } 12 \\
& \boldsymbol{T}_{i n, C V}=\frac{\boldsymbol{T}_{i n, \sigma}}{\boldsymbol{T}_{\text {in }, \mu}}=\mathbf{0 . 2 2 3} \quad \text { Eq. } 13
\end{aligned}
$$

Table 11. Average and Coefficients of Variation Used in the Thermal Sensitivity Analysis,

\begin{tabular}{|l|l|c|}
\hline \multicolumn{1}{|c|}{ Variable } & \multicolumn{1}{c|}{ Average, $\boldsymbol{\mu}$} & $\mathbf{C V}, \boldsymbol{\sigma} / \boldsymbol{\mu}$ \\
\hline Coolant Temperature (Tcool) & $324 \mathrm{~K}$ & 0.05 \\
\hline Fuel pellet LHGR (q) & $350 \mathrm{~W} / \mathrm{cm}$ & 0.05 \\
\hline Rodlet inside radius (r2) & $4.15 \mathrm{~mm}$ & 0.003 \\
\hline Rodlet outside radius (r3) & $4.75 \mathrm{~mm}$ & 0.002 \\
\hline Capsule inside radius (r4) & $4.78 \mathrm{~mm}$ & 0.005 \\
\hline Capsule outside radius (r5) & $5.49 \mathrm{~mm}$ & 0.005 \\
\hline $\begin{array}{l}\text { Rodlet thermal conductivity } \\
\text { (kC) }\end{array}$ & $16.8 \mathrm{~W} /(\mathrm{m} \mathrm{K})$ & 0.05 \\
\hline $\begin{array}{l}\text { Helium gap thermal } \\
\text { conductivity - rodlet to capsule } \\
\text { (kD) }\end{array}$ & $0.243 \mathrm{~W} /(\mathrm{m} \mathrm{K})$ & 0.05 \\
\hline $\begin{array}{l}\text { Capsule thermal conductivity } \\
\text { (kE) }\end{array}$ & $18.5 \mathrm{~W} /(\mathrm{m} \mathrm{K})$ & 0.05 \\
\hline $\begin{array}{l}\text { Heat transfer coefficient from } \\
\text { capsule to coolant (h) }\end{array}$ & $4.80 \times 10^{4} \mathrm{~W} /\left(\mathrm{m}^{2} \mathrm{~K}\right)$ & 0.05 \\
\hline
\end{tabular}


It is clear from the values above that there was the potential for a huge variation in temperature calculations. The variables responsible for this can be found by using the uncertainty percentage contribution, UPC, for each variable. This was calculated by using Eq. 4. These values are shown in Figure 12. As can be seen in Figure 12, the capsule inside radius (r4) was by far the largest contributors to the variation in the rodlet temperature. These two alone accounted for virtually all of the total variation in the cladding temperature calculations.

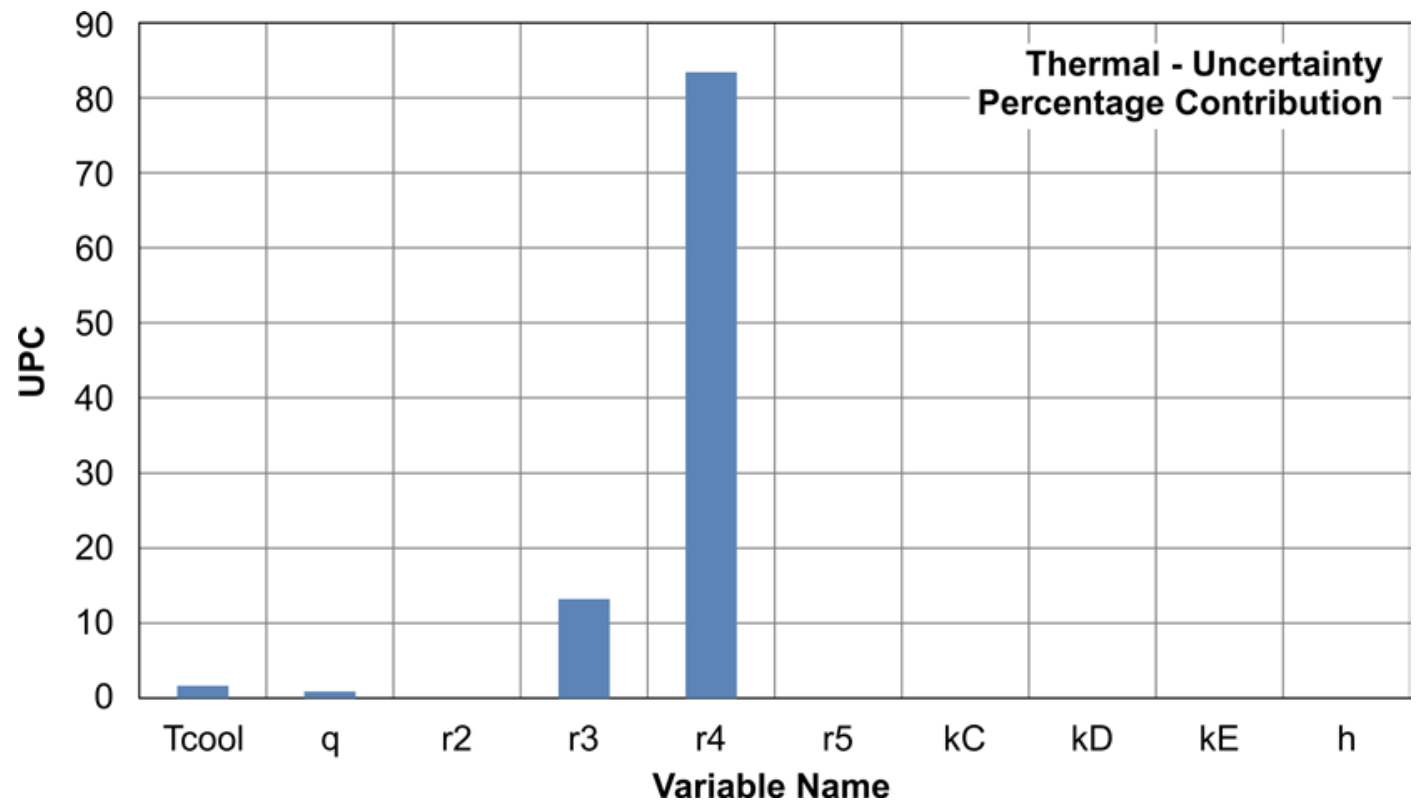

Figure 12. UPC results for thermal calculations.

Using Eq. 5, the uncertainty magnification factors, UMF, for each variable was calculated. These values are shown in Figure 13. The UMF values for the rodlet outside radius (r3) and capsule inside radius (r4) were the dominant contributors to the UMF. The UMF values for the other variables were below 1.0 indicating that their uncertainties were diminished as they propagated through the stress equation. 


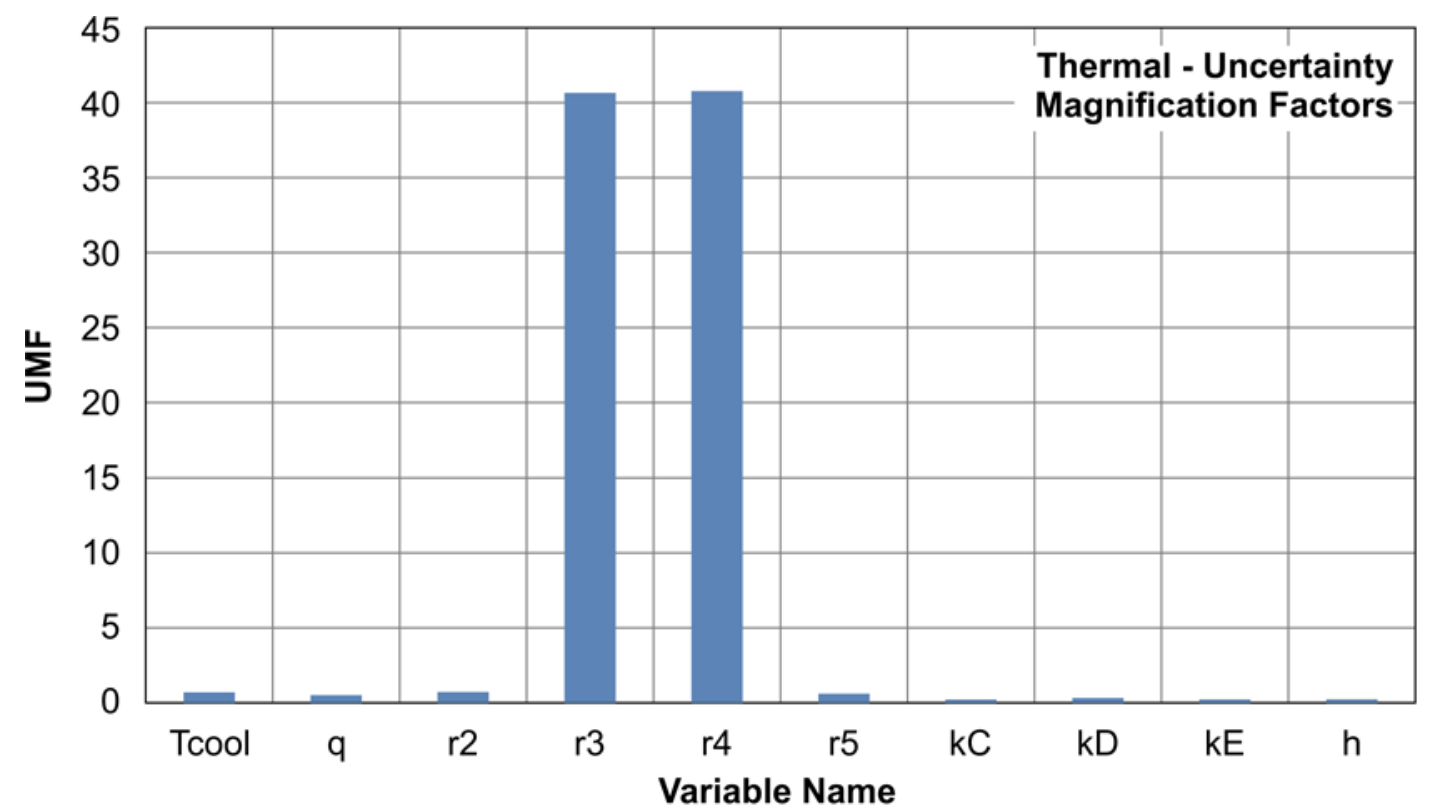

Figure 13. UMF results for thermal calculations.

Based on the thermal analysis, the dimensions defining the gap between the rodlet and capsule (rodlet outside radius and capsule inside radius) are the most important variables to control for providing accurate prediction of the rodlet temperatures. The remaining variables have relatively little effect.

\subsubsection{Structural Calculations}

Modeling the rodlet as a thick-walled cylinder with an internal pressure and steady-state temperature gradient, the effective stress at the inside surface is a function of the following variables [Eq. 14]:

$$
M s=M s(r 2, r 3, p, E, v, \alpha, \Delta T)
$$

Where:

$$
\begin{aligned}
& \text { Ms = Effective stress or von Mises stress } \\
& \text { r2 = Rodlet inside radius } \\
& \text { r3 = Rodlet outside radius } \\
& \mathrm{p}=\text { Rodlet internal pressure } \\
& \mathrm{E}=\text { Rodlet elastic modulus } \\
& \mathrm{v}=\text { Rodlet Poisson's ratio } \\
& \alpha=\text { Coefficient of linear thermal expansion } \\
& \Delta \mathrm{T}=\text { Temperature gradient through the wall }
\end{aligned}
$$

For this analysis, the average and coefficients of variation (CV) for each of the variables are shown in Table 12. 
Table 12. Average and Coefficients of Variation Used in the Structural Sensitivity Analysis.

\begin{tabular}{|l|c|c|}
\hline \multicolumn{1}{|c|}{ Variable } & Average, $\boldsymbol{\mu}$ & $\mathbf{C V}, \boldsymbol{\sigma} / \boldsymbol{\mu}$ \\
\hline \hline Rodlet inside radius (r2) & $4.15 \mathrm{~mm}$ & 0.003 \\
\hline Rodlet outside radius (r3) & $4.75 \mathrm{~mm}$ & 0.002 \\
\hline Internal pressure (p) & $12.2 \mathrm{MPa}$ & 0.328 \\
\hline Rodlet elastic modulus (E) & $77.9 \mathrm{Gpa}$ & 0.05 \\
\hline Rodlet Poisson's ratio (v) & 0.26 & 0.05 \\
\hline Thermal Expansion $(\alpha)$ & $6.62 \times 10^{-6} \mathrm{~m} / \mathrm{m} / \mathrm{K}$ & 0.05 \\
\hline $\begin{array}{l}\text { Temperature gradient } \\
(\Delta \mathrm{T})\end{array}$ & $62.2 \mathrm{~K}$ & 0.223 \\
\hline
\end{tabular}

The internal pressure and temperature gradient, as well as their calculated $\mathbf{C V}, \boldsymbol{\sigma} / \boldsymbol{\mu}$ values, come from the neutronics and thermal analysis, respectively. Note that the elastic modulus and thermal expansion coefficient are functions of temperature. For this analysis, average temperature through the wall thickness was used to determine their values. Using equations 2 and 3, the resulting average, standard deviation, and coefficient of variation for the effective stress are [Eq. 15, Eq. 16, and Eq. 17]:

$$
\begin{array}{lc}
M \boldsymbol{s}_{\boldsymbol{\mu}}=\mathbf{7 1 . 6} \boldsymbol{M P a} & \text { Eq. } 15 \\
\boldsymbol{M} \boldsymbol{s}_{\boldsymbol{\sigma}}=\mathbf{2 9 . 6} \boldsymbol{M P a} & \text { Eq. } 16 \\
\boldsymbol{M} \boldsymbol{s}_{\boldsymbol{C V}}=\frac{\boldsymbol{M} \boldsymbol{s}_{\sigma}}{\boldsymbol{M} \boldsymbol{s}_{\boldsymbol{\mu}}}=\mathbf{0 . 4 1 4} & \text { Eq. } 17
\end{array}
$$

Using Eq. 5, the uncertainty percentage contribution, UPC, for each variable can be calculated. These values are shown in Figure 14. As can be seen in Figure 14, the internal pressure (p) was by far the biggest contributors to the variation in the effective stress. This factor alone accounted for over $80 \%$ of the total variation in the effective stress.

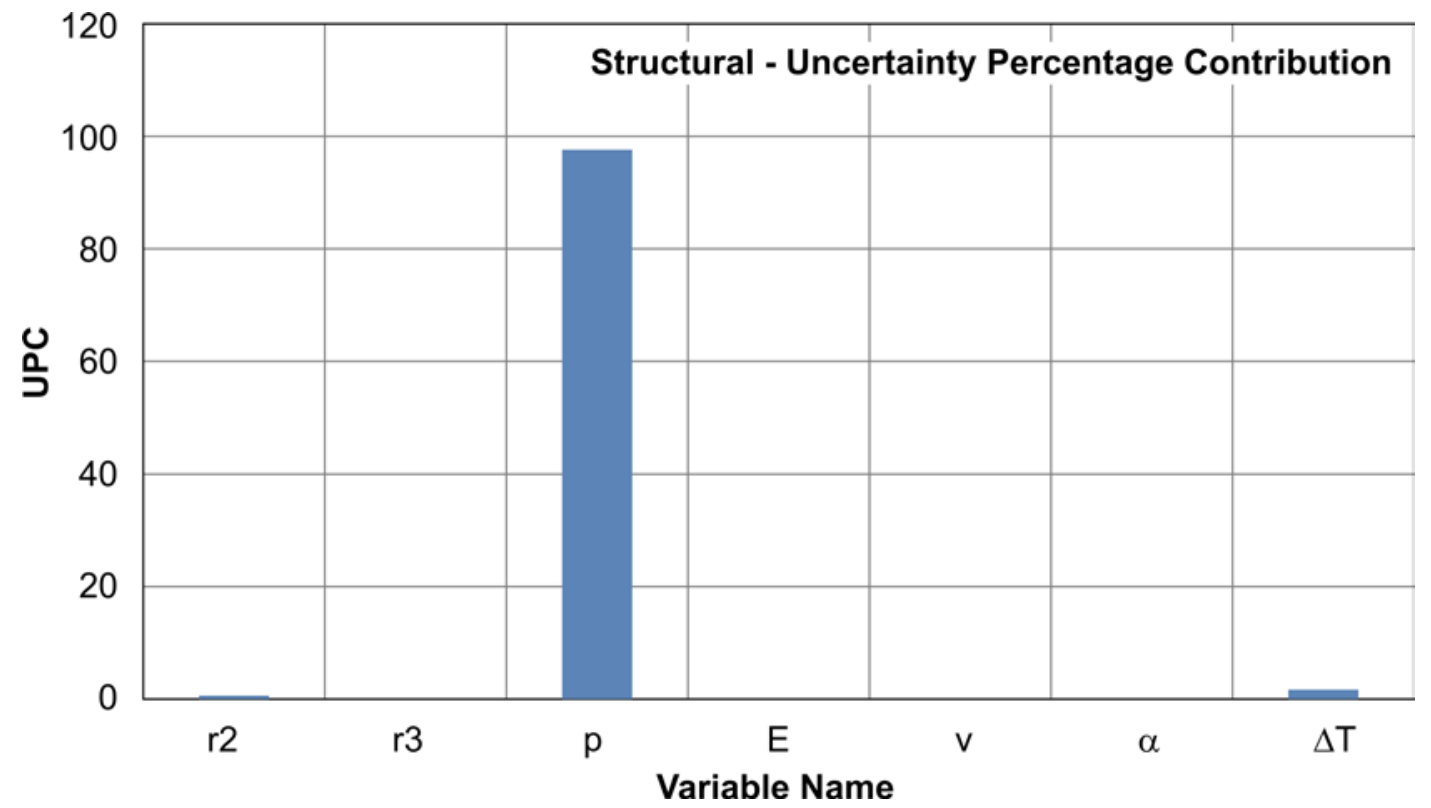

Figure 14. UPC results for stress calculations. 
Using Eq. 4, the uncertainty magnification factors, UMF, for each variable can be calculated. These values are shown in Figure 15 The UMF values for the inside radius, outside radius, and internal pressure are all over 1.0 indicating that their uncertainties are magnified as they propagate through the stress equation. The UMF values for the other variables are below 1.0 indicating that their uncertainties were diminished as they propagated through the stress equation.

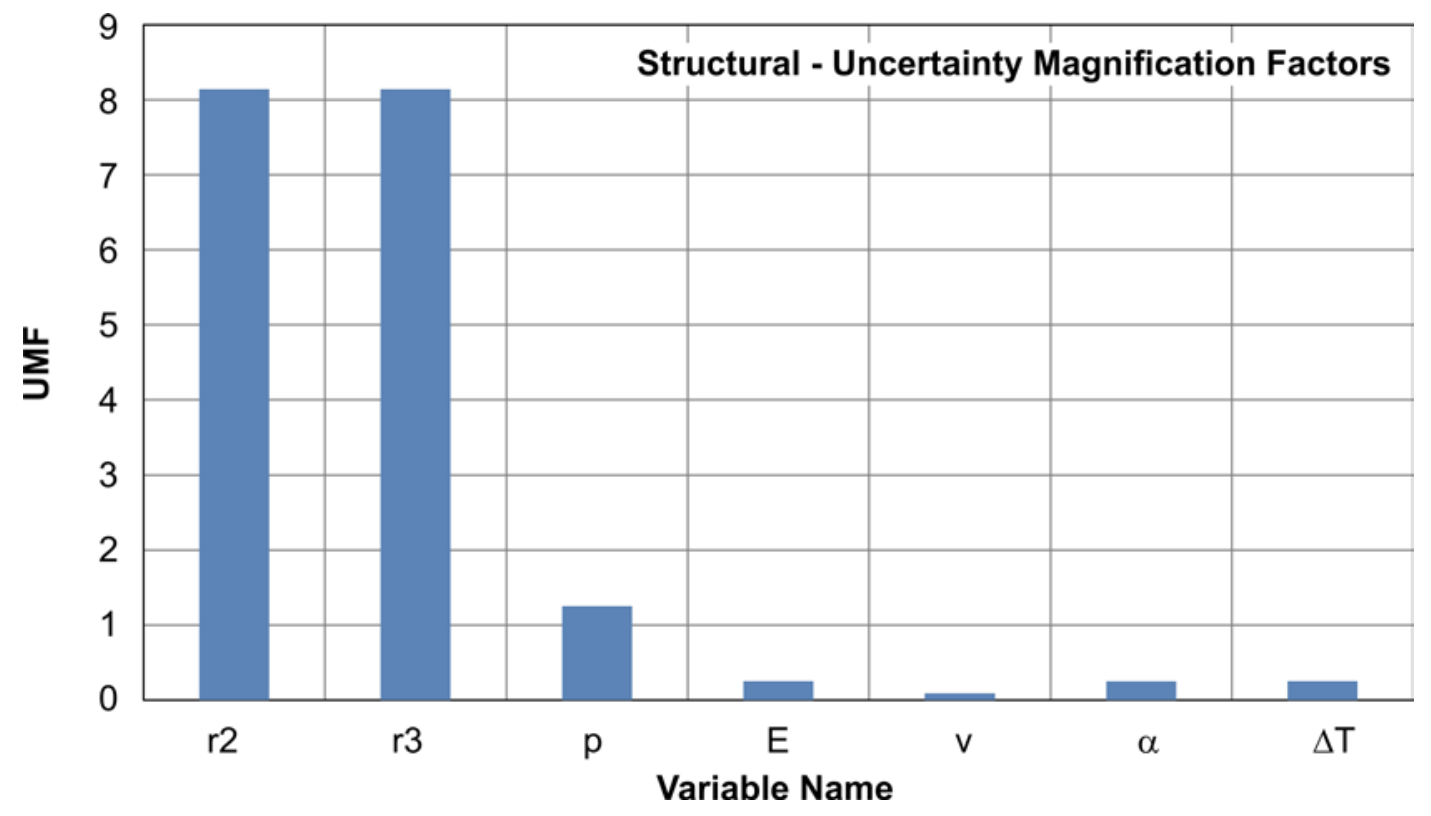

Figure 15. UMC results for stress calculations.

Based on this structural sensitivity analysis, the internal pressure is the largest contributor to uncertainty in rodlet stress. As fission gas release, which is unknown for many new fuels, is the largest contributor to pressure uncertainty, a conservative approach must be taken to assure that rodlet stresses do not lead to breaching of the rodlet.

\section{FABRICATION CHALLENGES}

Fabrication of ATF concept experiment assemblies will typically involve non-traditional materials not previously fabricated using traditional procedures and equipment. ATF concept development teams should provide the fabricators as much information about the material properties and behavior observed in prototype testing, mock-up fabrication and assembly, and chemical and mechanical testing of the materials that may have been performed in the concept development phase. Special handling, machining, welding, and inspection of materials, if known, should also be communicated to the experiment fabrication and inspection team. In many instances, fabrication, welding, and inspections of innovative materials is an art rather than a skill. The more information the fabrication and inspection team has a priori, the more successful they will be in fabricating and inspecting the experiment.

The Taylor Series Method parameter sensitivity analysis shows that the rodlet dimensional tolerance was by far the most critical BOL variable for calculating clad temperature. The ABAQUS clad temperature sensitivity analysis showed that a 0.001 inch change in the capsule/rodlet gas gap (a function of rodlet and capsule dimensional tolerance) resulted in approximately a $+/-20 \%$ change in cladding temperature. This demonstrated that the rodlet and capsule dimensional tolerances have to be as small as possible (close to $0.0013 \mathrm{~cm}$ [0.0005 inches]) and the capsule/rodlet pairs must be individually selected to meet the gas gap dimension as close to nominal as possible. High precision machining and inspection equipment was needed to meet this requirement. For the condition in which rodlet tubes were machined using traditional lathes, very high precision inspection equipment was needed, such as a Coordinate Measuring Machine, CMM (Figure 16), to ensure that the tube dimensions were within the drawing tolerance both axially and azimuthally. 

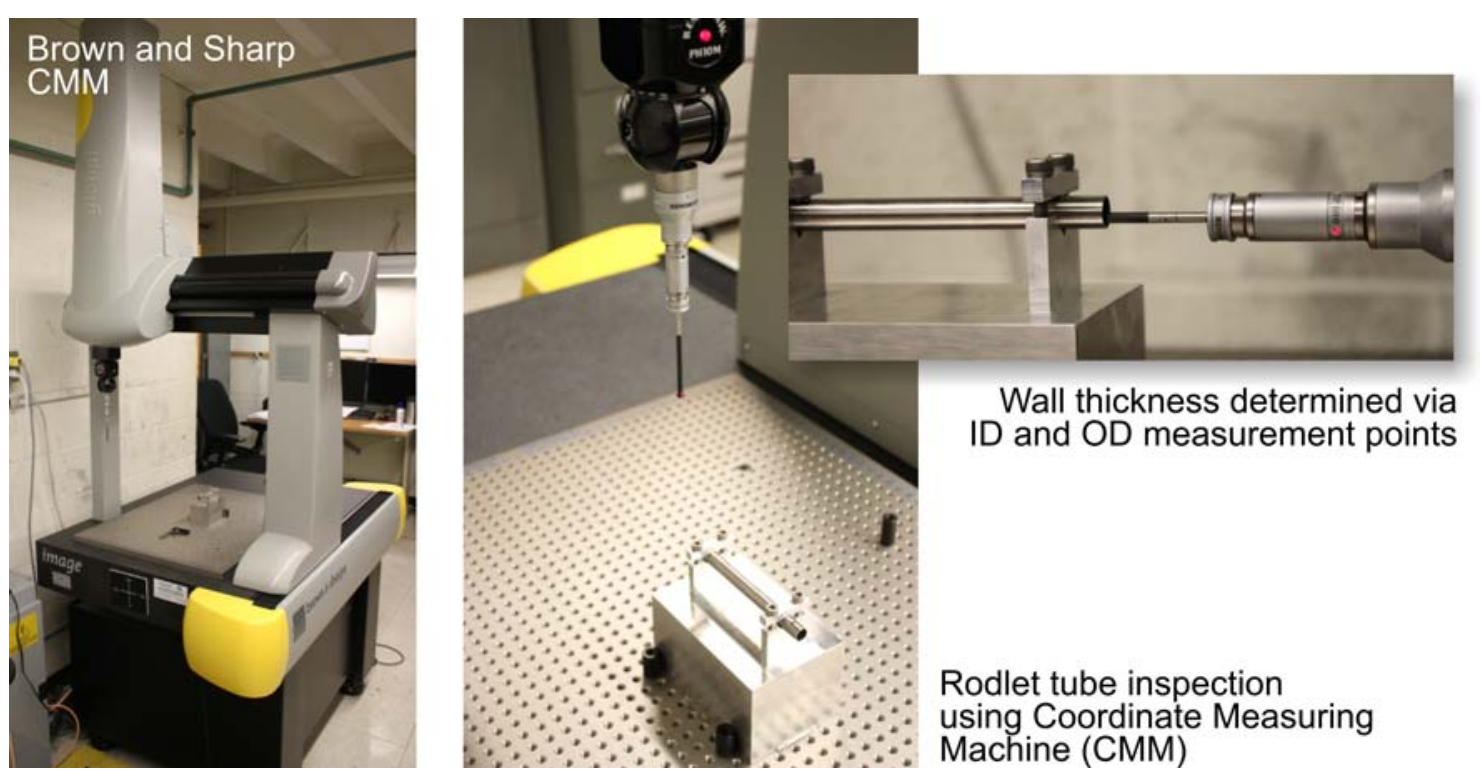

Figure 16. CMM used to measure axial and azimuthal dimensions of the capsule and rodlet tube I.D. and O.D.

\subsection{As-Built Analysis}

BISON was used to produce 3D temperature profiles of an APMT clad rodlet to investigate the impact on cladding temperature resulting from variation in cladding diameter and thickness as a result of tube gun-drilling fabrication techniques. BISON is a finite element nuclear fuel performance code that is built using INL's Multiphysics Object-oriented Simulation Environment (MOOSE), a parallel, finite element-based framework to solve systems of coupled non-linear partial differential equations using the Jacobian-free Newton Krylov (JFNK) method. The BISON governing relations currently consist of fully coupled partial differential equations for energy, species, and momentum conservation [add reference here]. The energy and momentum equations (used for thermomechanics analysis) were used for this simulation [7]. As-built axial and radial dimensions are measured on the rodlet tube using a CMM. The CMM data were directly imported into the BISON model and 3D temperature profiles were calculated. Figure 17 and Figure 18 show the BISON results. The as-built 3D temperature profiles were then be used to determine if the rodlet clad temperatures (axial and azimuthal) were with the desired test range for the ATF concept design. 


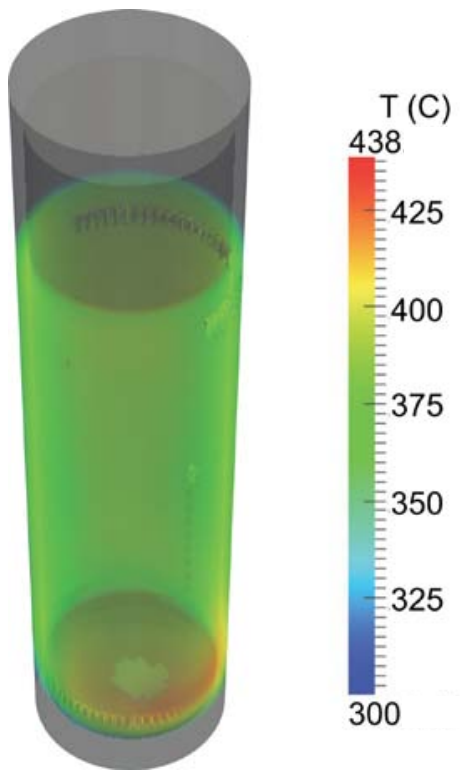

Figure 17: Volumetric view of temperature with a threshold of $300^{\circ} \mathrm{C}-450^{\circ} \mathrm{C}$.

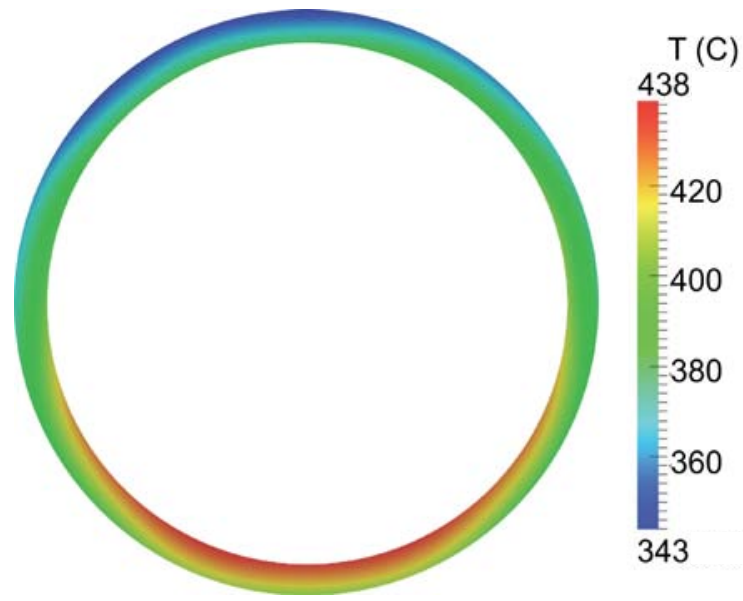

Figure 18: Radial slice of temperature in the cladding at a height of 0.125 inches.

\section{CONCLUSIONS}

Design, analysis, and fabrication processes for ATR drop-in capsule experiment preparation are presented in this paper to demonstrate the importance of special design considerations, parameter sensitivity analysis, and precise fabrication and inspection techniques for innovative materials used in Accident Tolerant Fuels experiment assemblies.

A Taylor Series Method sensitivity analysis approach was used to identify the most critical variables in cladding and rodlet stress, temperature, and pressure calculations for design analyses. The results showed that internal rodlet pressure calculations are most sensitive to the fission gas release rate uncertainty while temperature calculations are most sensitive to cladding and capsule I.D. and O.D. dimensional uncertainties. The analysis showed that stress calculations are most sensitive to rodlet internal pressure uncertainties, however the results also indicated that the inside radius, outside radius, and internal pressure were all magnified as they propagate through the stress equation. 
Although experiment component parameters are also sensitive to changes in reactor lobe power and uncertainties in fuel fission gas release rate during irradiation, lobe power cannot always be controlled in a test reactor (in particular, the ATR) and the fission gas release rate during irradiation testing is part of the experiment (i.e., data is not available). In contrast, the experiment component fabrication and inspection can be controlled and should be performed using much more precise equipment than traditionally used.

The authors recognize that irradiation-induced properties such as creep, thermal expansion, swelling, and densification also contribute to fuel system performance, however the objective of the ATF experiments is to test innovative materials to quantify these unknown properties. This paper describes an initial attempt to identify fuel system variables that design engineers can focus on controlling as close to nominal as possible in the fabrication and irradiation testing of the ATF fuel system concepts. Future work is planned to evaluate and/or validate the results presented in the paper using the fuel performance code BISON.

This paper demonstrates the importance for ATF concept development teams to provide the fabricators as much information about the material properties and behavior observed in prototype testing, mock-up fabrication and assembly, and chemical and mechanical testing of the materials that may have been performed in the concept development phase. Special handling, machining, welding, and inspection of materials, if known, should also be communicated to the experiment fabrication and inspection team. In many instances, fabrication, welding, and inspections of innovative materials is an art rather than a skill. The more information the fabrication and inspection team has a priori, the more successful they will be in fabricating and inspecting the experiment. 


\section{REFERENCES}

1. Bragg-Sitton, S.M., Development of advanced accident-tolerant fuels for commercial LWRs, Nuclear News, March 2014, pp. 83-91 (2014).

2. Irradiation Testing of Accident Tolerant Fuels in the ATR: The ATF-1 Test Series, PLN-4444, Idaho National Laboratory, April 2013.

3. Advanced Fuels Campaign Light Water Reactor Accident Tolerant Fuel Performance Metrics, INL/EXT-13-29957, U.S. DOE Office of Nuclear Energy, February 2014.

4. L. J. Ott and R. N. Morris, "Irradiation Tests of Mixed-Oxide Fuel Prepared with Weapons-Derived Plutonium", Journal of Nuclear Materials, Vol. 371 (2007) pp.314-328.

5. H. W. Coleman and W. G. Steele, Experimentation, Validation, and Uncertainty Analysis for Engineers, $3^{\text {rd }}$ Edition, 2009.

6. R. M. Brach and P. F. Dunn, Uncertainty Analysis for Forensic Analysis, $2^{\text {nd }}$ Edition, 2009.

7. R. L. Williamson, J. D. Hales, S. R. Novascone, M. R. Tonks, D. R. Gaston, C. J. Permann, D. Andrs, and R. C. Martineau. Multidimensional multiphysics simulation of nuclear fuel behavior. Journal of Nuclear Materials 423:149-163, 2012. 


\section{ACKNOWLDEGEMENTS}

The development of this paper was jointly supported by the U.S. Department of Energy (DOE) Fuel Cycle Research and Development (FCRD) and the Light Water Reactor Sustainability (LWRS) programs. The ATF irradiation testing is funded by the FCRD Program. Acknowledgements for support of the ATF experiments at the INL go to the following:

$\begin{array}{ll}\text { Shannon Bragg-Sitton } & \text { FCRD LWR Fuel Coordination Lead } \\ \text { Heather Chichester } & \text { FCRD Irradiation Testing Technical Lead } \\ \text { Steven Hayes } & \text { ATF Fuel Performance Principal Investigator } \\ \text { Jason Harp } & \text { ATF Fuel Performance and PIE Principal Investigator } \\ \text { Glenn Moore } & \text { ATF Fabrication Principal Investigator } \\ \text { Michael Teague } & \text { AFC Lead Design Engineer and Thermal Analyst } \\ \text { Brian Durtschi } & \text { Design Engineer } \\ \text { Misti Lillo } & \text { Physics Analyst } \\ \text { Christopher Glass } & \text { Physics Analyst } \\ \text { Glenn Roth } & \text { Thermal Analyst } \\ \text { Warren Jones } & \text { Thermal Analyst } \\ \text { Kelly Ellis } & \text { Structural Analyst } \\ \text { Jesse Johns } & \text { BISON Analyst } \\ \text { Pavel Medvedev } & \text { BISON Analyst } \\ \text { Stephen Evans } & \text { ATR Representative } \\ \text { Michael David } & \text { ATR Experiment Engineer } \\ \text { Christine White } & \text { Graphic Artist }\end{array}$



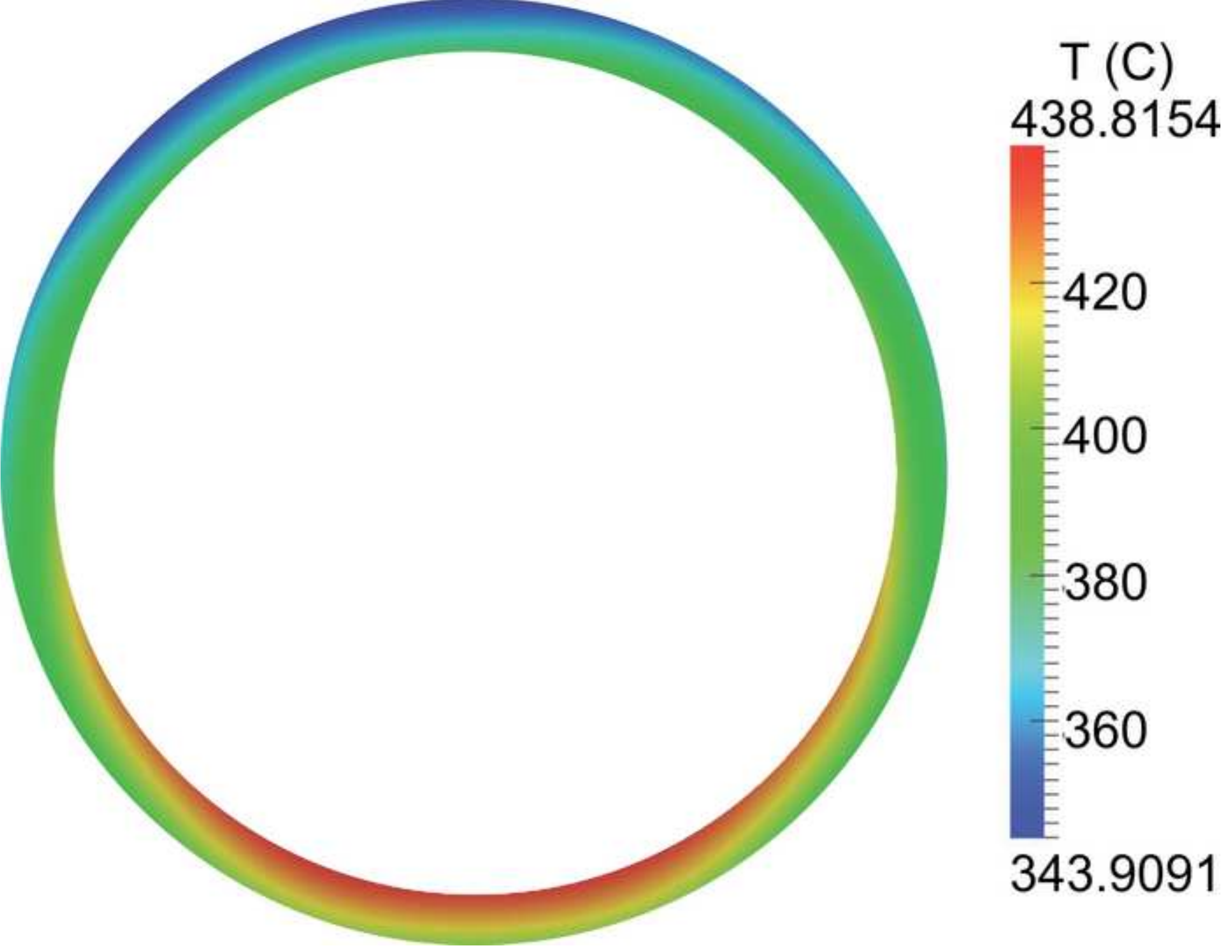
Figure 17

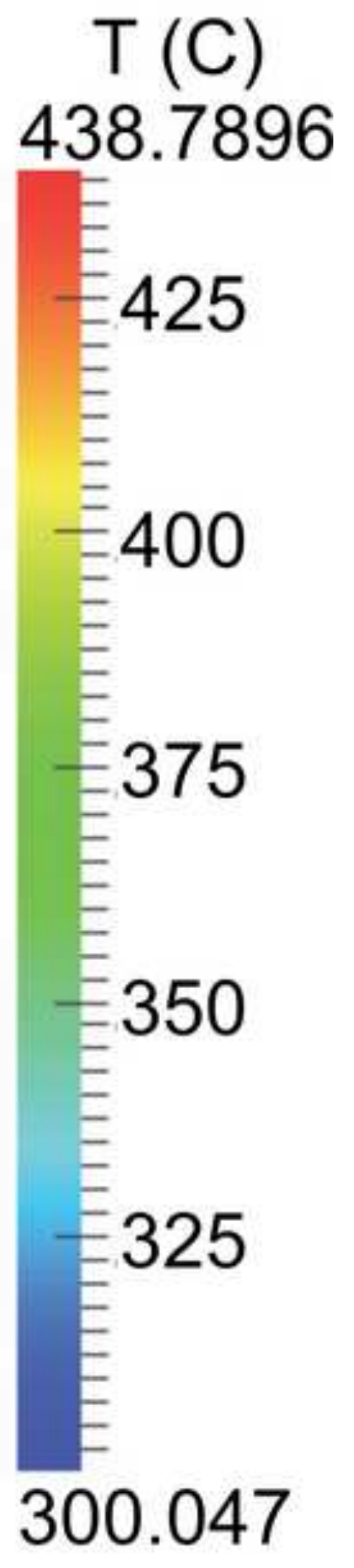



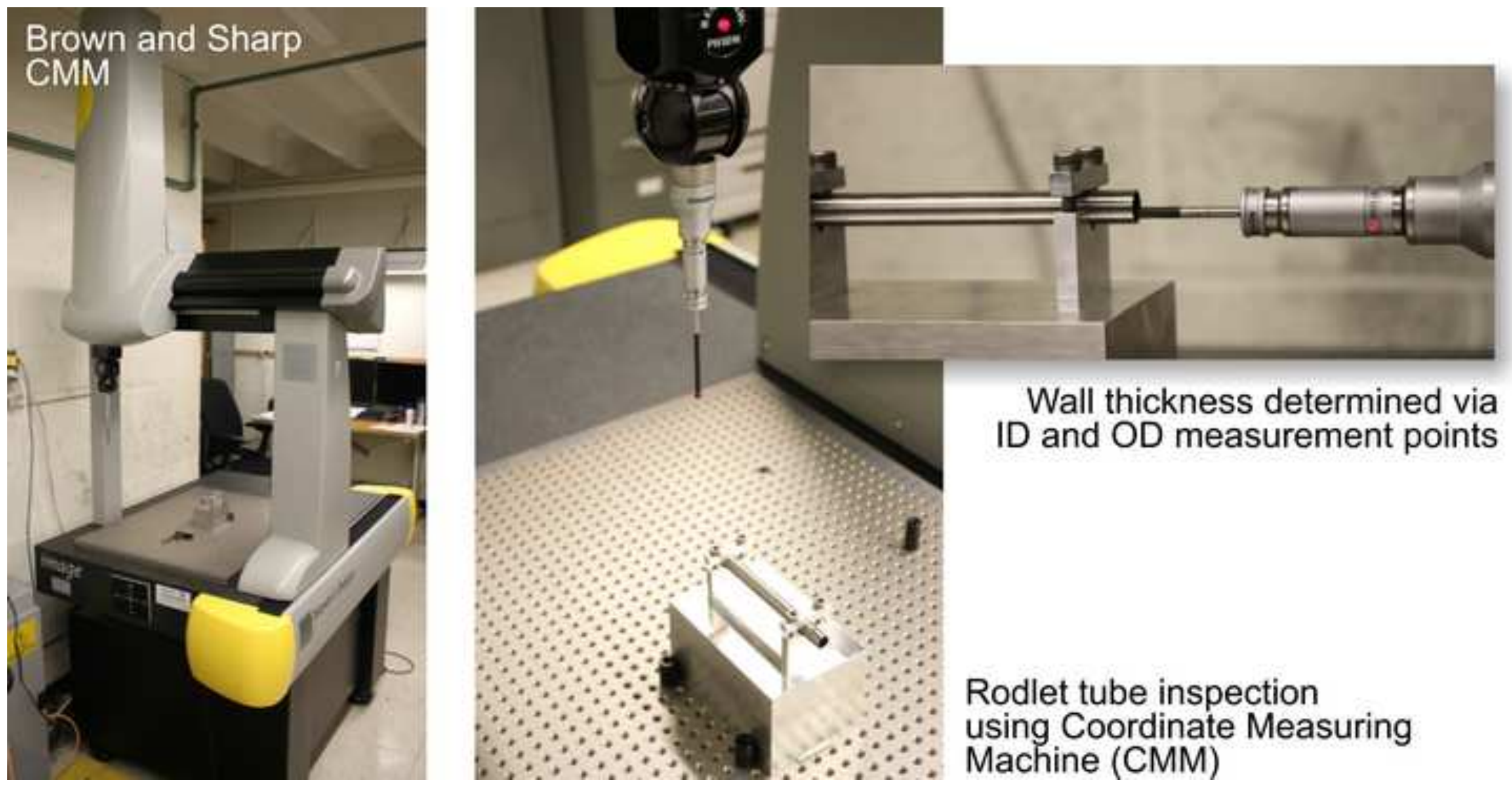

Rodlet tube inspection using Coordinate Measuring Machine (CMM) 


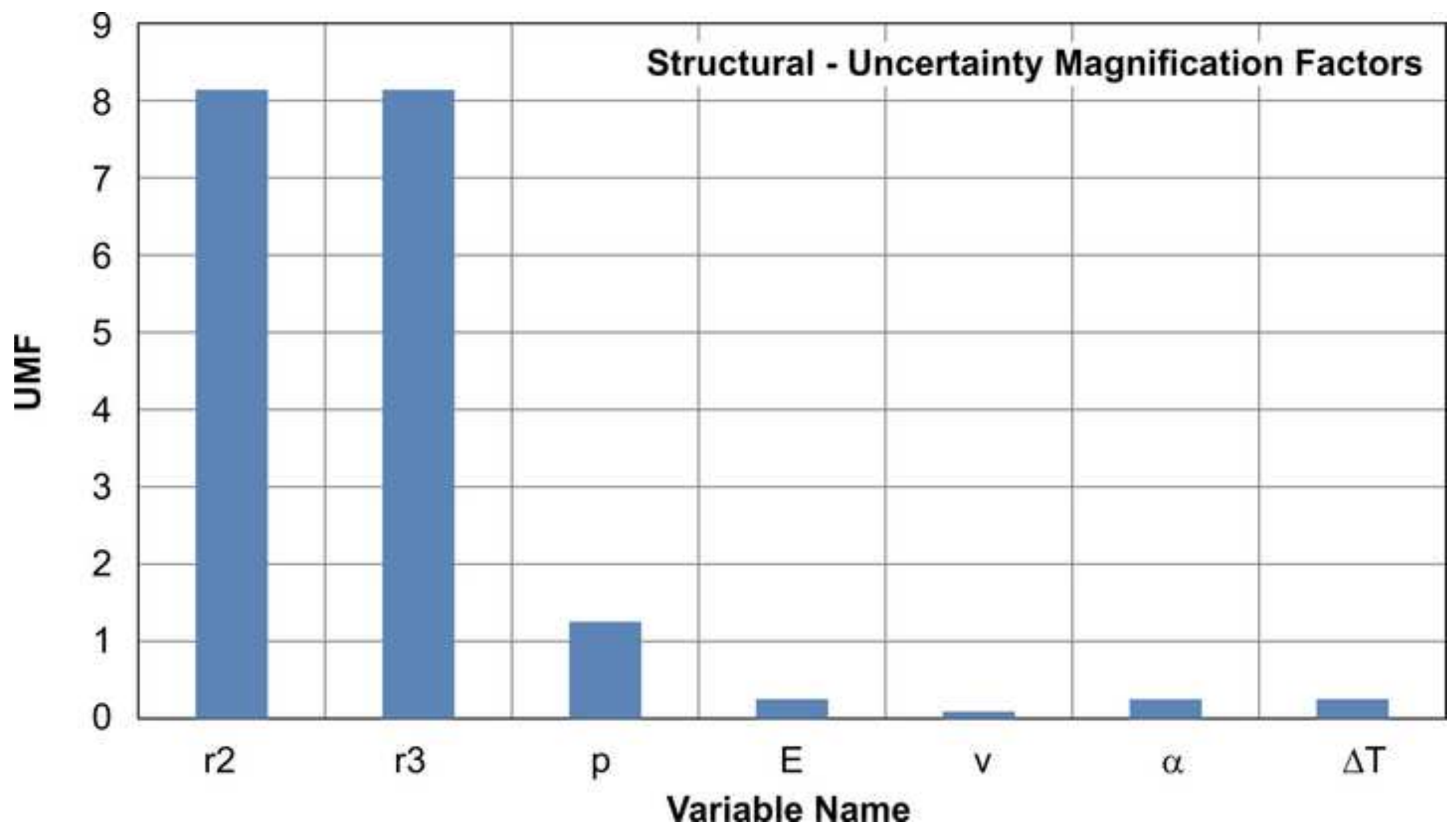




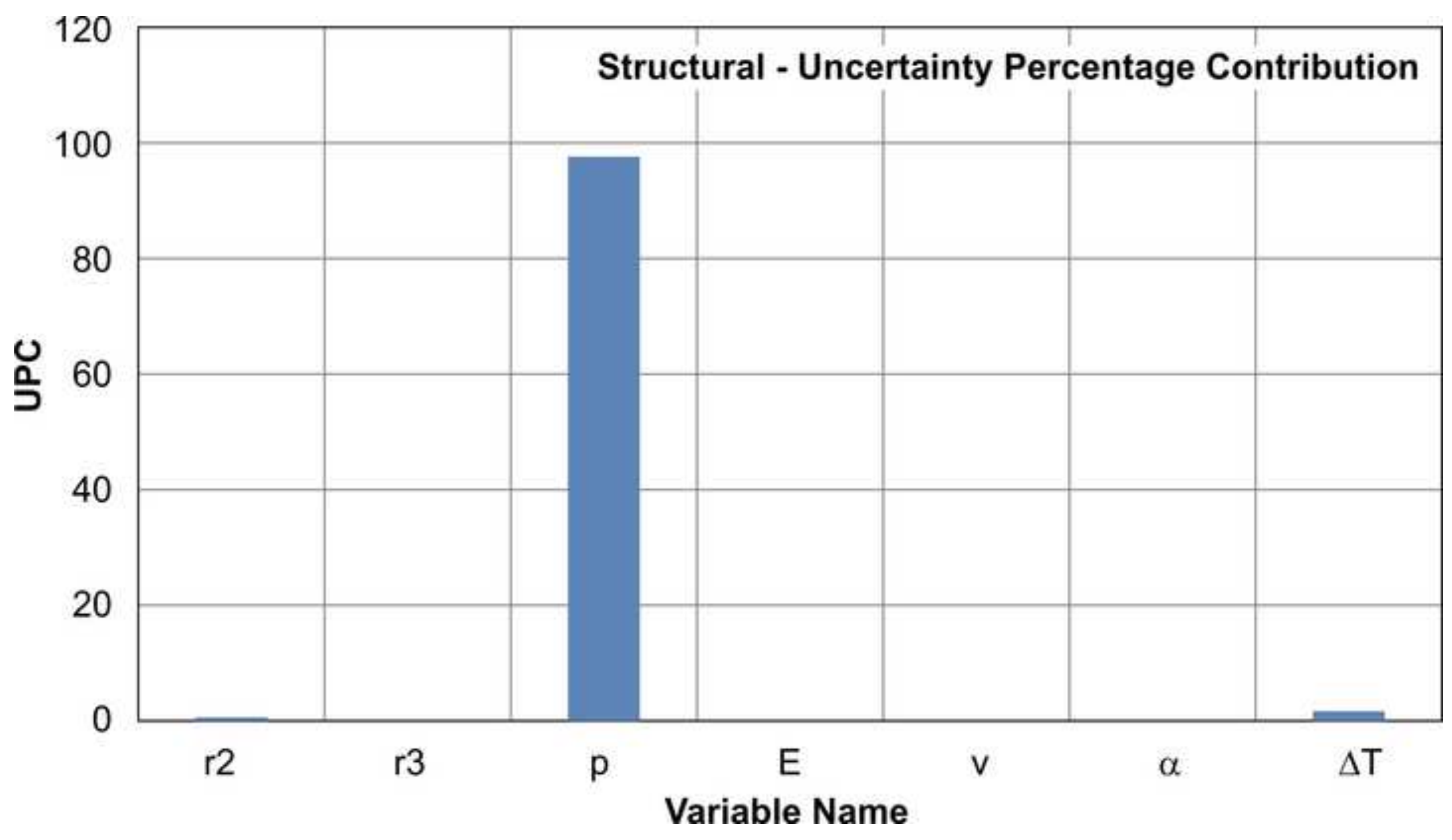




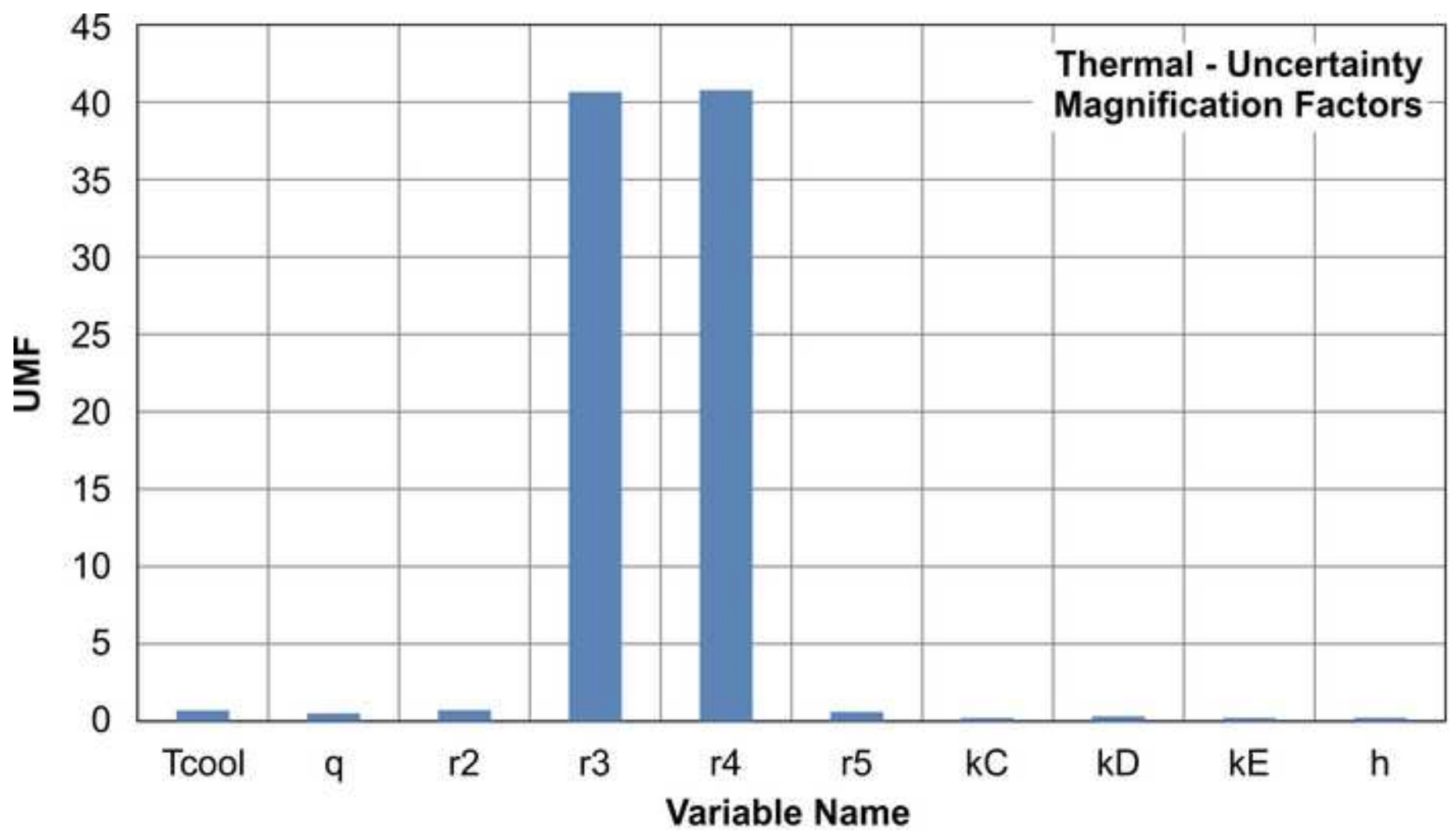




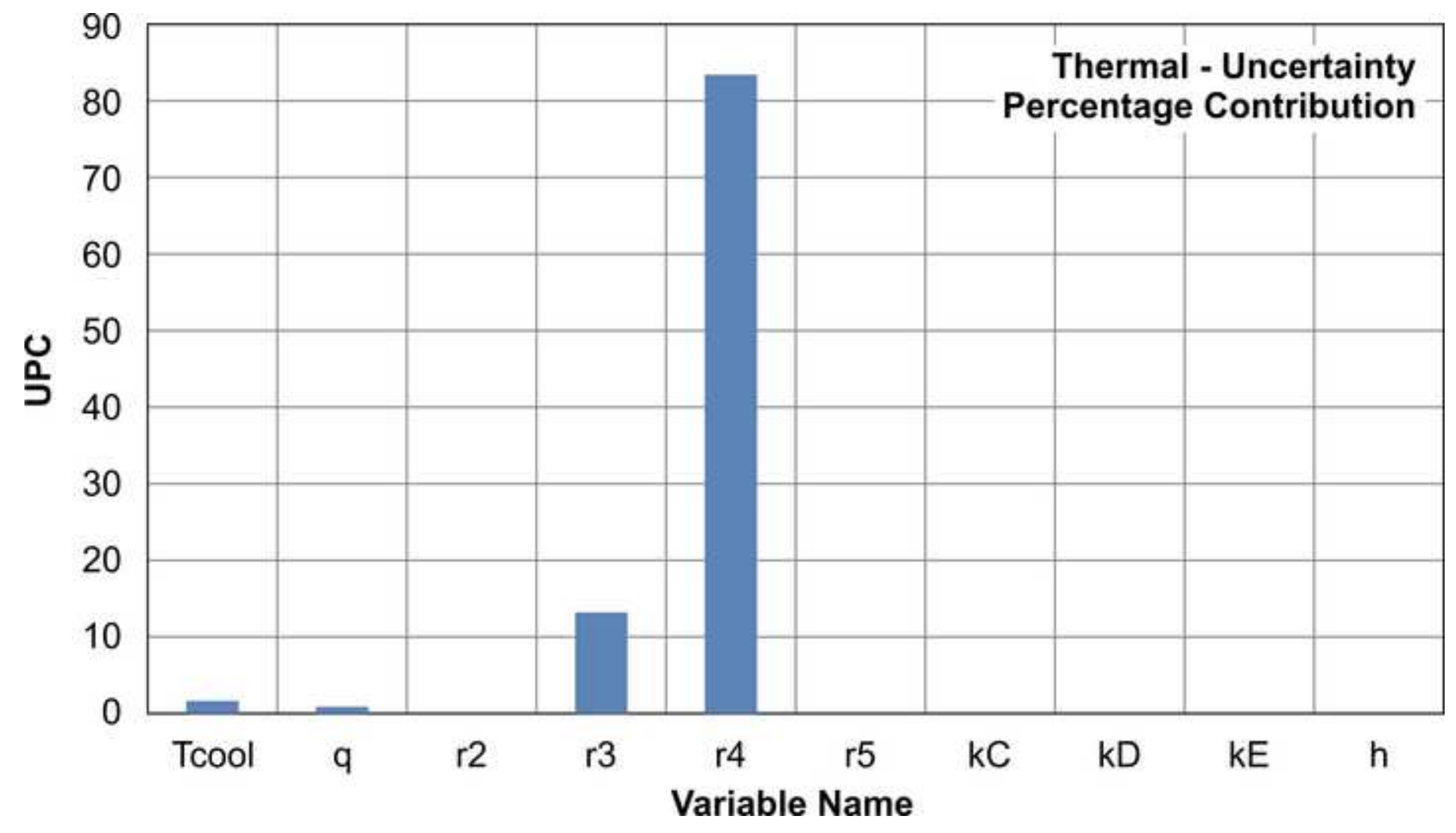




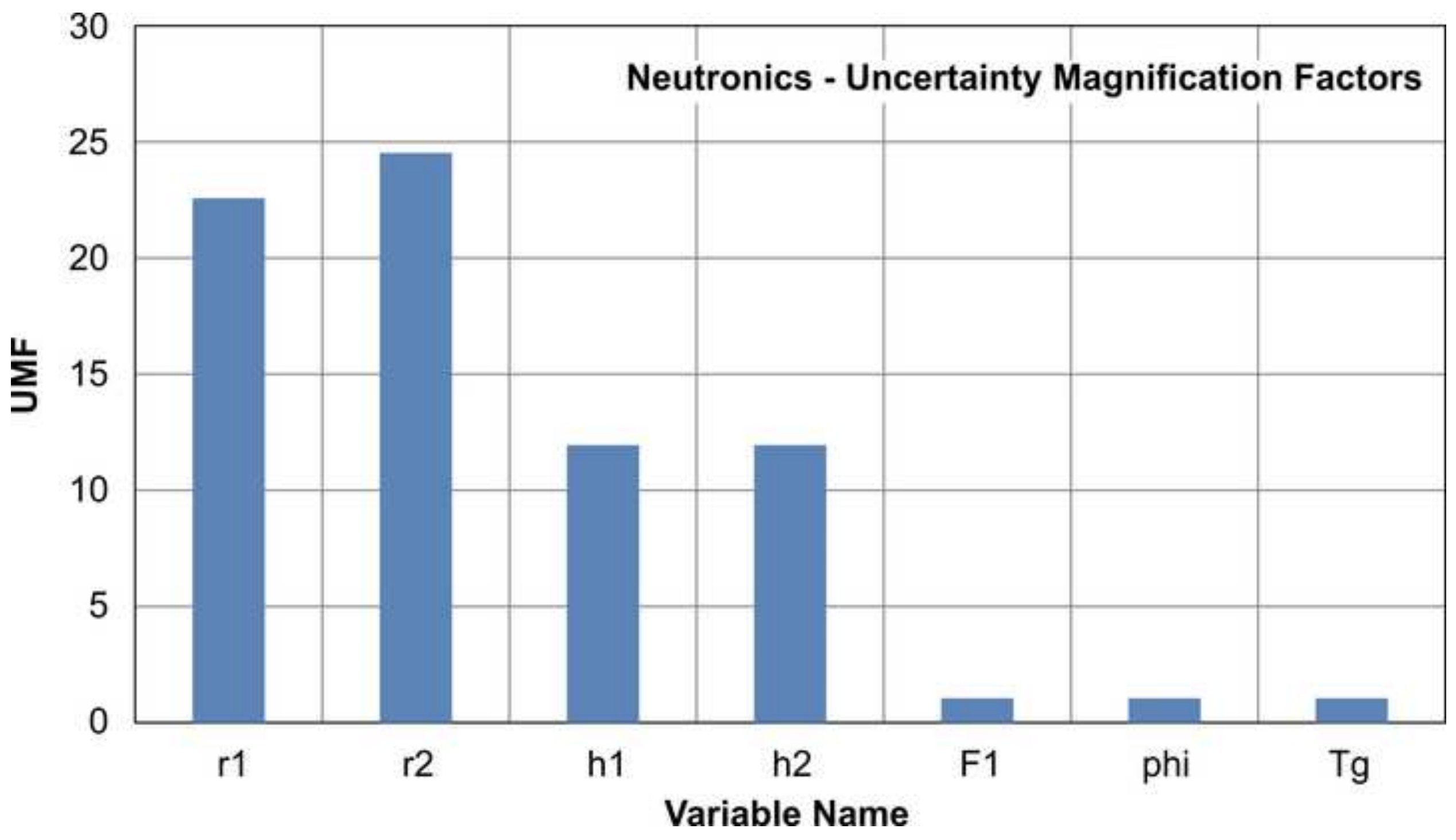




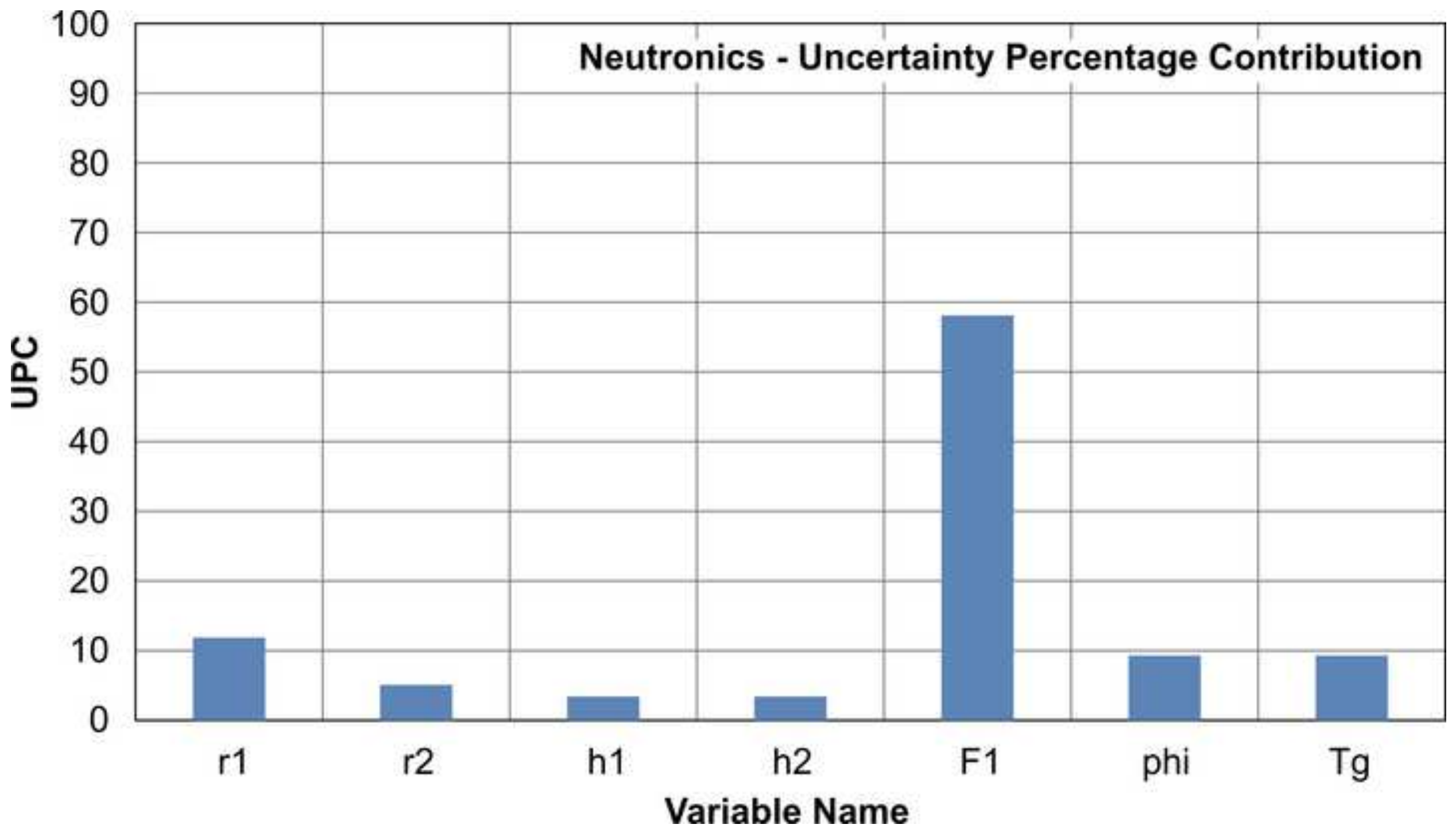


Figure 9

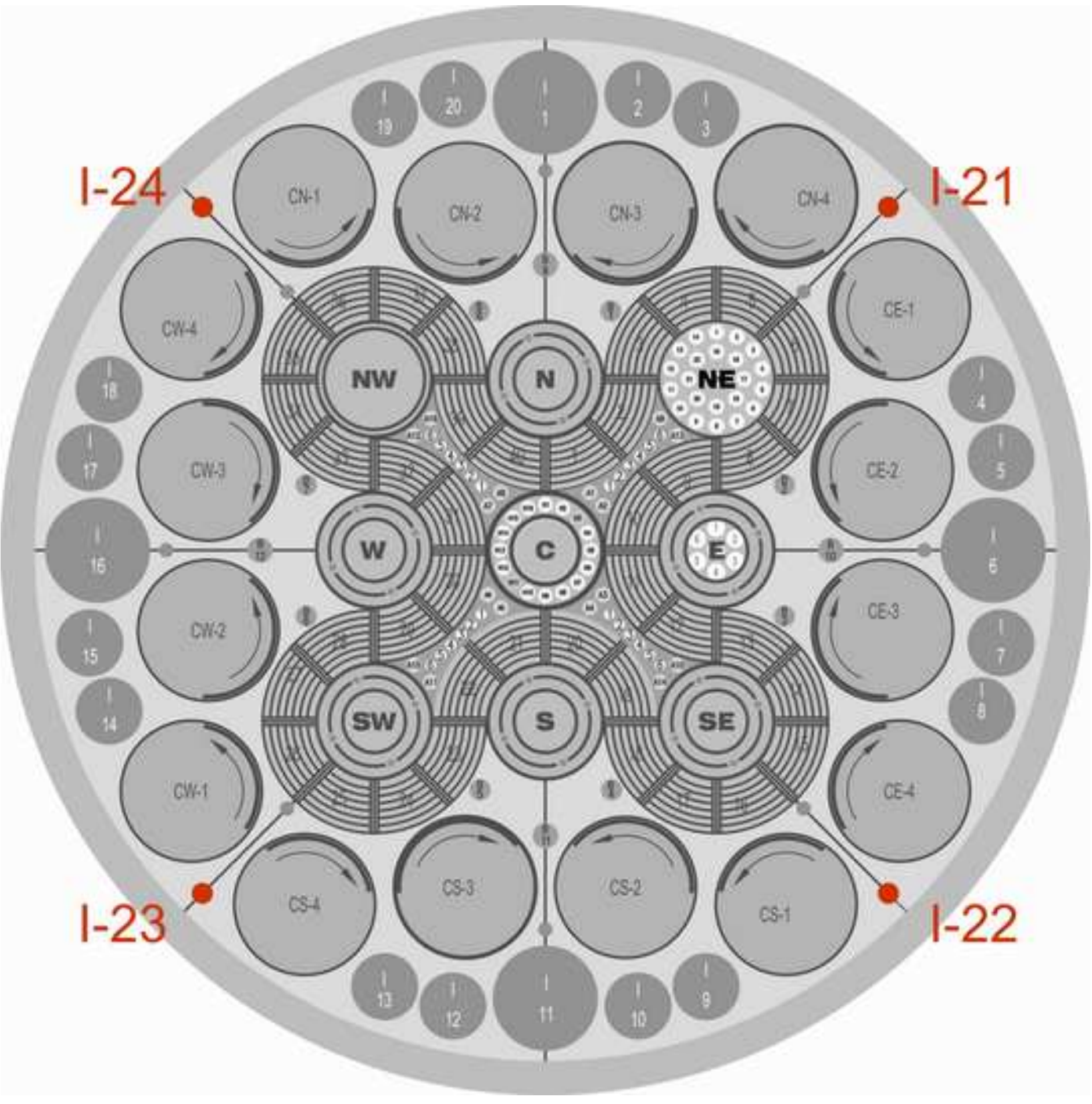




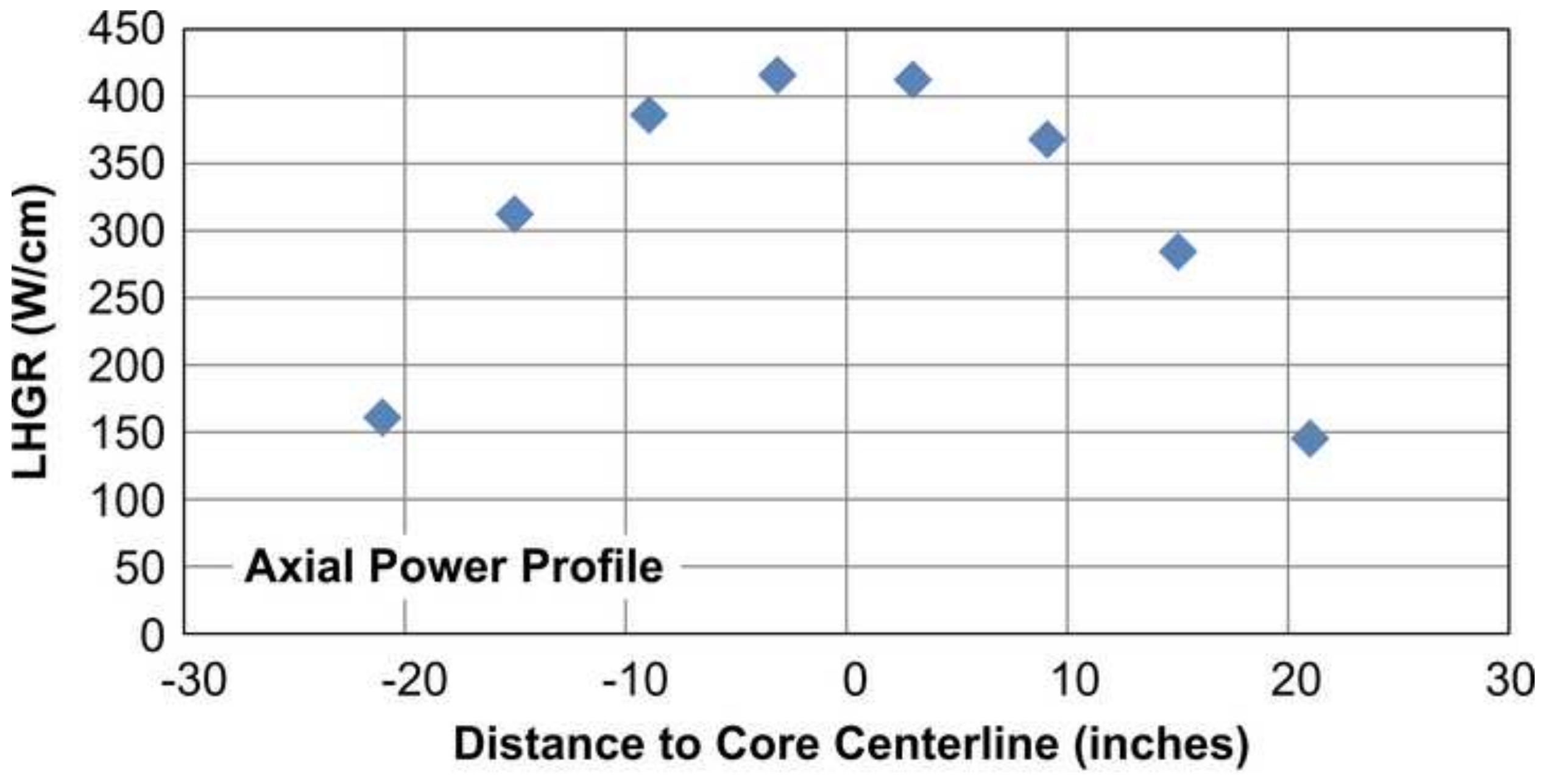


Figure 2

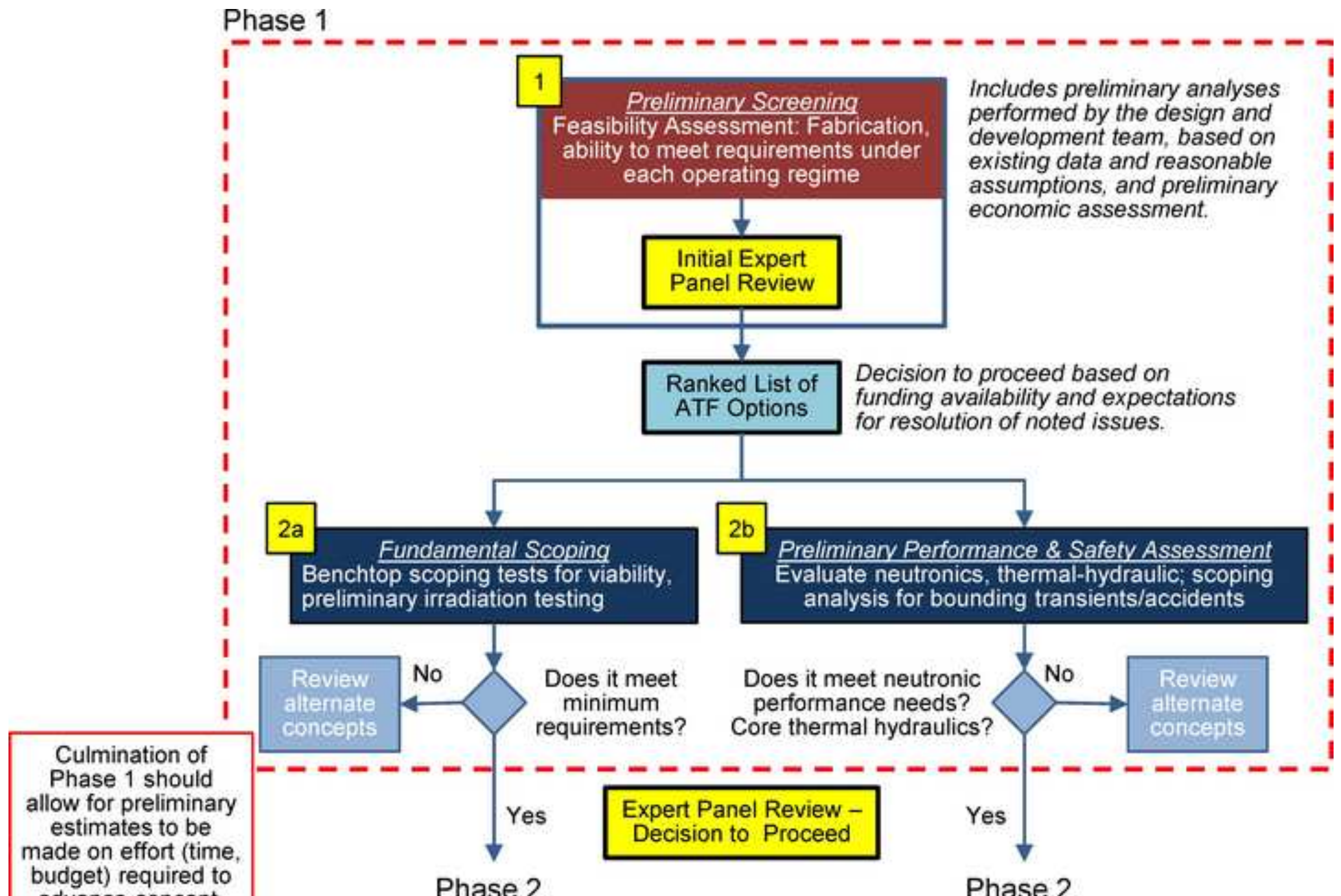

dget) required to advance concept.

Phase 2

Phase 2 


\section{ATF Test Capsule Design Nominal Dimensions}

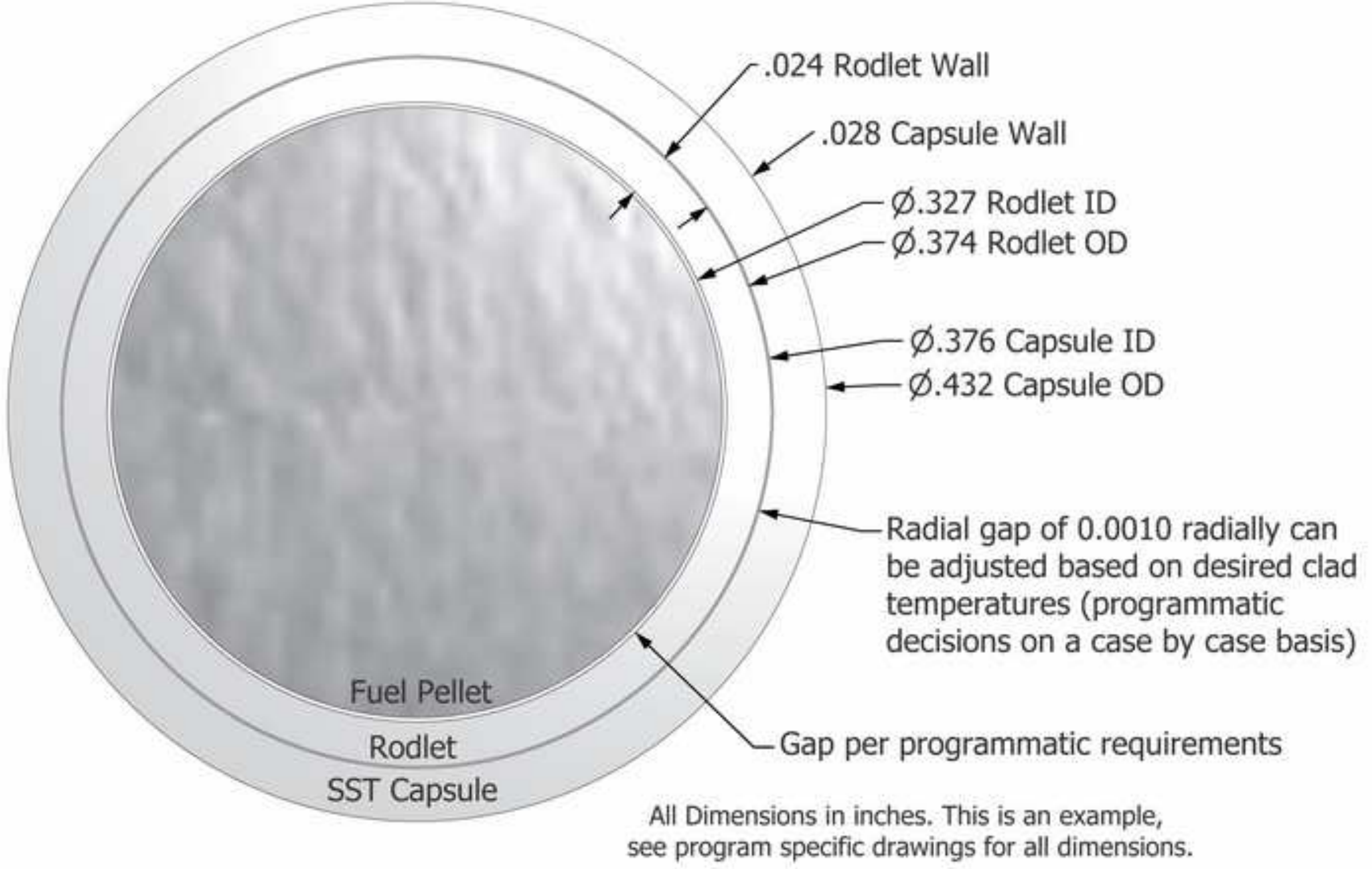


Figure 7

3 Positions

21 Total Capsules

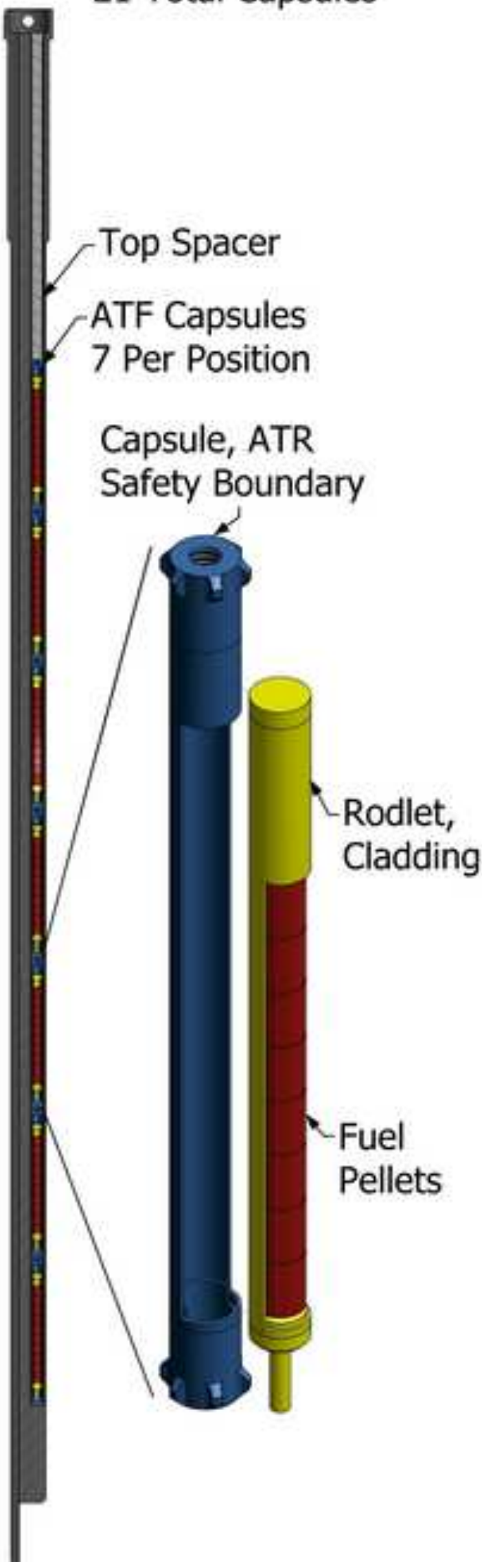


Figure 3
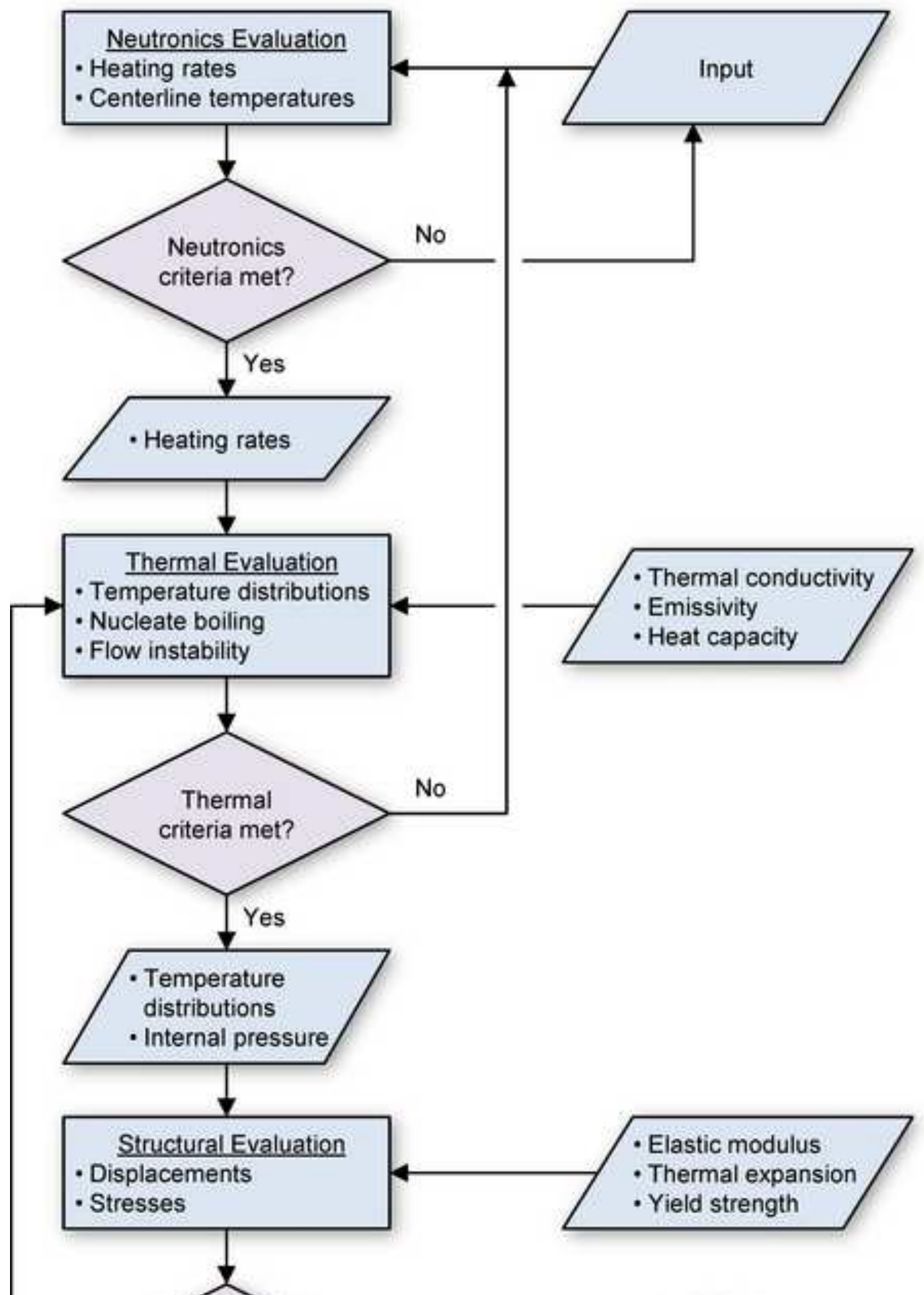

No

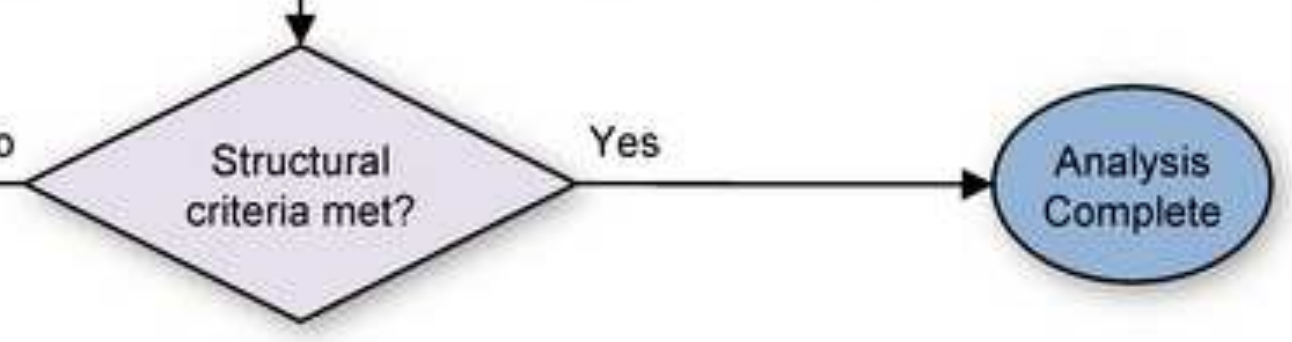




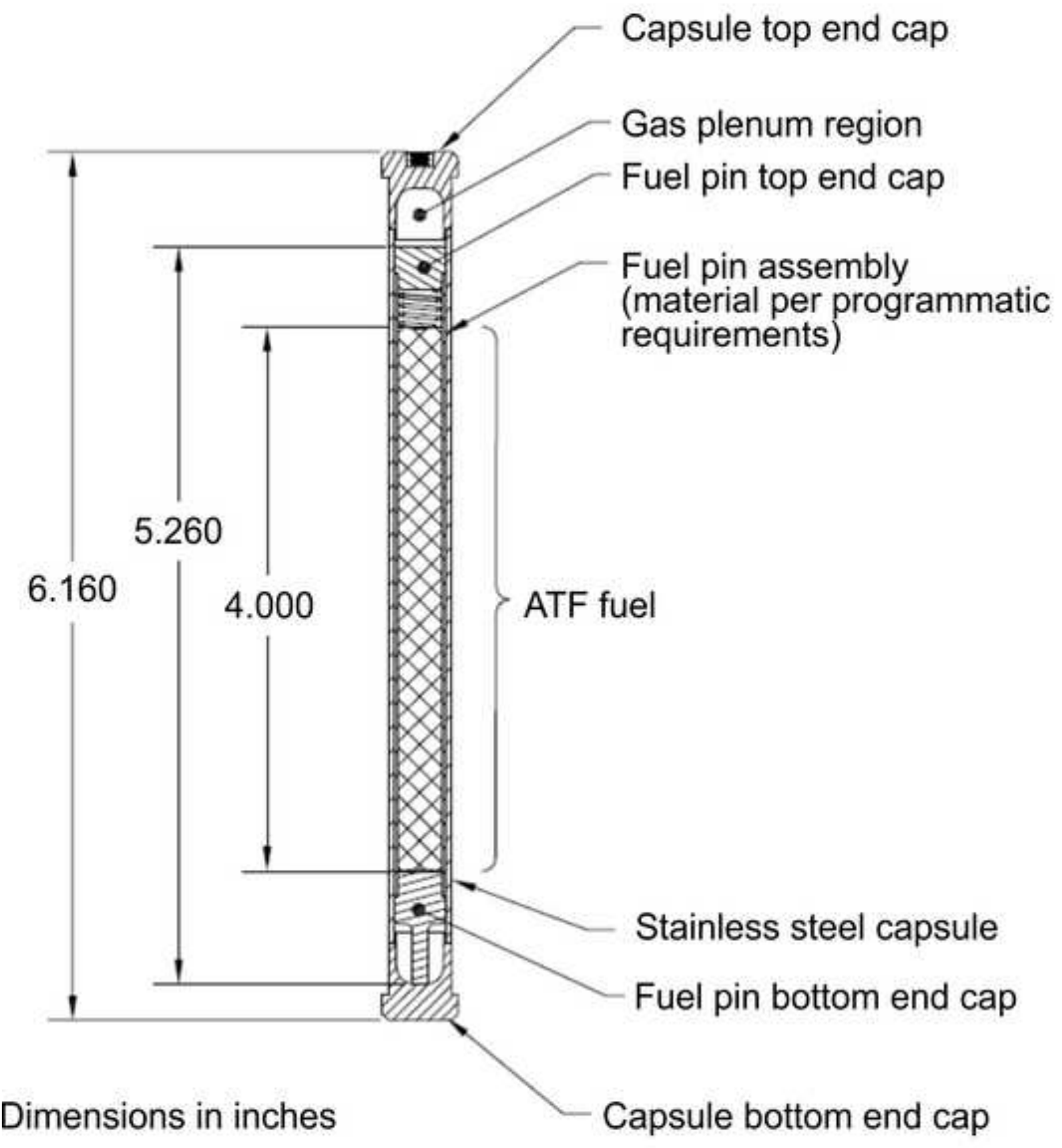




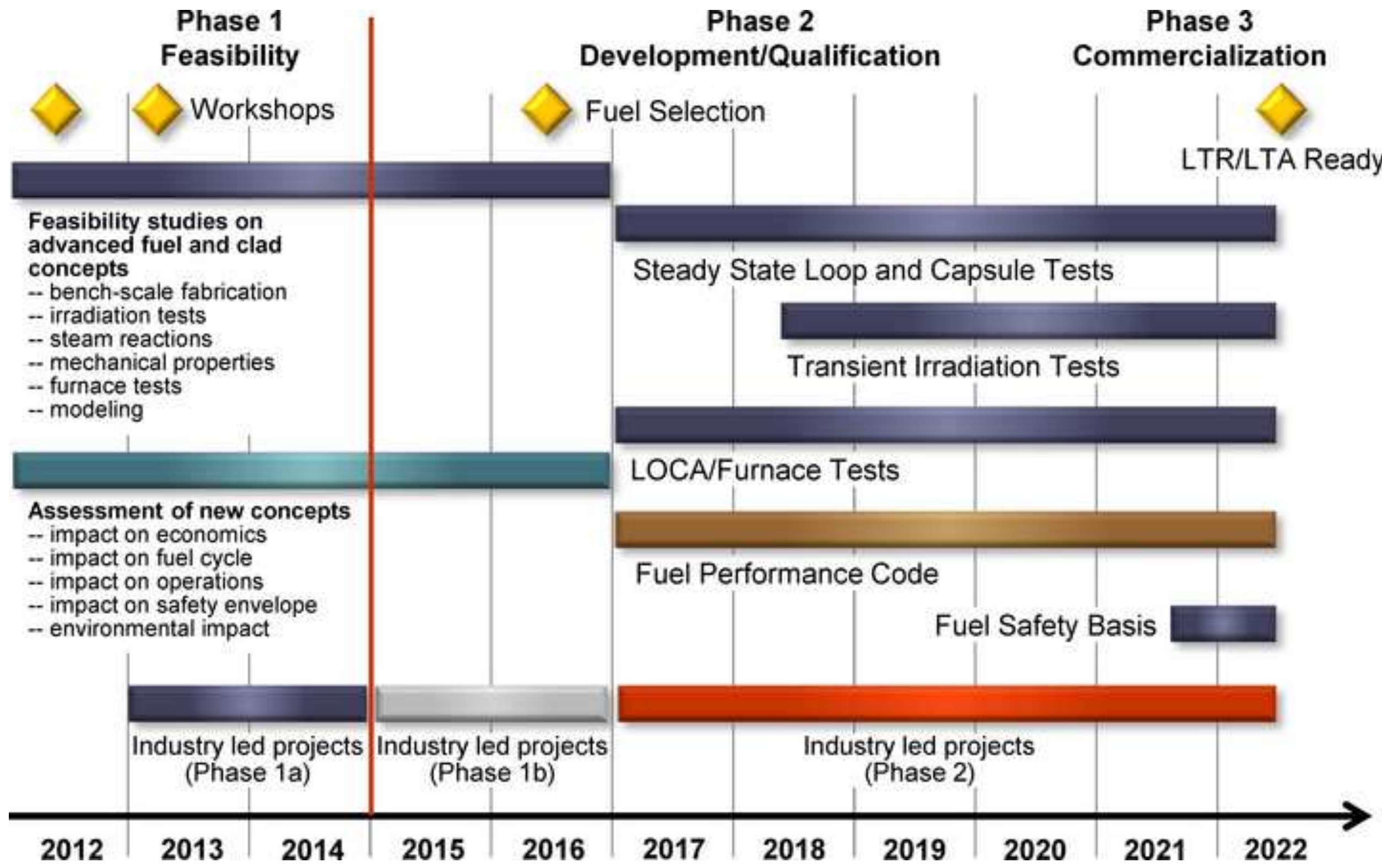




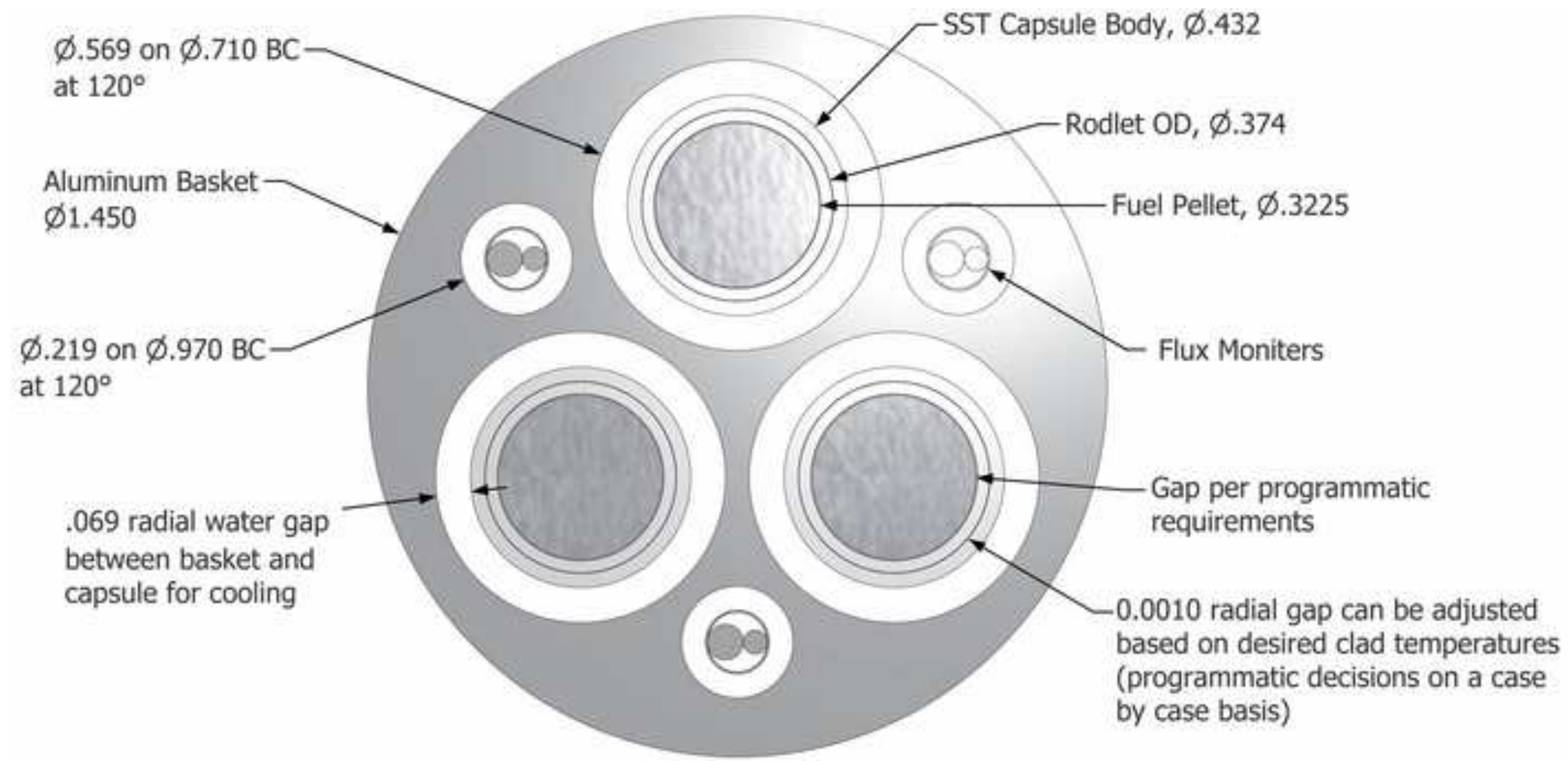

All Dimensions in inches. This is an example, see program specific drawings for all dimensions. 